\title{
Enhancing antibody folding and secretion by tailoring the Saccharomyces cerevisiae endoplasmic reticulum
}

\author{
Jorg C. de Ruijter ${ }^{\dagger}$, Essi V. Koskela ${ }^{\dagger}$ and Alexander D. Frey ${ }^{*}$
}

\begin{abstract}
Background: The yeast Saccharomyces cerevisiae provides intriguing possibilities for synthetic biology and bioprocess applications, but its use is still constrained by cellular characteristics that limit the product yields. Considering the production of advanced biopharmaceuticals, a major hindrance lies in the yeast endoplasmic reticulum (ER), as it is not equipped for efficient and large scale folding of complex proteins, such as human antibodies.

Results: Following the example of professional secretory cells, we show that inducing an ER expansion in yeast by deleting the lipid-regulator gene OP/1 can improve the secretion capacity of full-length antibodies up to fourfold. Based on wild-type and ER-enlarged yeast strains, we conducted a screening of a folding factor overexpression library to identify proteins and their expression levels that enhance the secretion of antibodies. Out of six genes tested, addition of the peptidyl-prolyl isomerase CPR5 provided the most beneficial effect on specific product yield while PDI1, ERO1, KAR2, LHS1 and SIL1 had a mild or even negative effect to antibody secretion efficiency. Combining genes for ER enhancement did not induce any significant additional effect compared to addition of just one element. By combining the $\triangle$ opi1 strain, with the enlarged ER, with CPR5 overexpression, we were able to boost the specific antibody product yield by a factor of 10 relative to the non-engineered strain.
\end{abstract}

Conclusions: Engineering protein folding in vivo is a major task for biopharmaceuticals production in yeast and needs to be optimized at several levels. By rational strain design and high-throughput screening applications we were able to increase the specific secreted antibody yields of S. cerevisiae up to 10-fold, providing a promising strain for further process optimization and platform development for antibody production.

Keywords: Protein folding, Heterologous protein production, Endoplasmic reticulum, Antibody, Chaperones

\section{Background}

The yeast Saccharomyces cerevisiae has proved its value in industrial applications, such as small chemicals and biofuel production, and is an excellent choice for new biotechnological processes due to the wide range of techniques to manipulate and cultivate the organism efficiently [1]. S. cerevisiae is also a popular choice for production of recombinant proteins and is currently the chosen production host for one-fifth of approved biopharmaceuticals on the market [2]. One of the main

\footnotetext{
*Correspondence: alexander.frey@aalto.fi

${ }^{\dagger}$ Jorg C. de Ruijter and Essi V. Koskela contributed equally to this paper Department of Biotechnology and Chemical Technology, Aalto University, Kemistintie 1, 02150 Espoo, Finland
}

limitations of producing recombinant pharmaceuticals in yeast is the different $\mathrm{N}$-glycan pattern compared to mammalian expression platforms [2]. However, producing correctly folded and glycosylated proteins for therapeutic applications in S. cerevisiae is about to become possible with the advancements of glycoengineered strains [3, 4]. With further optimization of the host and the production process, the yeast has great potential to become a viable platform to produce competitive yields of complex, functional mammalian target proteins, such as antibodies.

The yeast endoplasmic reticulum (ER) is limited in its recombinant protein folding capacity, and is thus an important target for cell engineering to improve product yields $[5,6]$. Producing recombinant proteins in yeast 
often leads to accumulation of folding intermediates in the ER and consequently to induction of the unfolded protein response (UPR) [7]. The UPR is a transcriptional program for concomitant upregulation of over 300 genes, including the upregulation of basal expression levels of constitutively expressed quality control components in the ER, which include folding factors, chaperones and the ER associated degradation (ERAD) machinery [8-10]. Inactivation of the UPR has shown to be detrimental for heterologous protein production [11, 12]. Although the UPR response is shown to be specific to the source of stress [13], heterologous protein yields could benefit most from a protein specific, fine-tuned expression of necessary helper proteins. One of the effects of UPR activation is also the Ino2/4 dependent induction of lipid biosynthesis genes, which play a key role in phospholipid biosynthesis required for membranes [14]. Although UPR leads to ER expansion, in yeast it has been shown that increasing ER size alone is sufficient to alleviate conformational stress independently from chaperone levels and UPR-induction [15]. Importance of ER size in effective protein secretion can also be noted from professional secretory cells, such as plasma cells and thyrocytes, that are characterized by an expanded ER that occupies most of the cytoplasmic space [16-19]. Although the development of secretory cells employs some of the same molecular routes as the UPR and has similar effects on gene expression, the onset of protein production is preceded by ER expansion [20]. Thus increasing the size of ER seems like a sensible strategy to preempt the negative effects of protein overexpression stress and the concomitant induction of UPR.

The limited folding rate in the ER of $S$. cerevisiae becomes a critical factor when producing large and complex proteins, such as human antibodies. In their native production environment, plasma cells, antibodies employ several classes of folding factors in their maturing process [21]. Molecular chaperones, such as BiP and its cochaperones, and folding enzymes, like protein disulfide isomerases (PDIs) and peptidyl-prolyl-isomerases (PPIase), all interact in a sequential manner with the folding light and heavy chain polypeptides to produce the secreted, mature heterotetrameric antibody [21, 22]. Although the yeast ER contains folding factors from all these classes, it is questionable whether the levels and stoichiometry of the proteins are adequate for efficient IgG-assembly. Different folding assistants co-operate with each other and form an extensive interaction network, possibly even a multiprotein complex [23-25]. Addition of just Pdi1p and/or Kar2p to enhance folding has been a popular approach [6,26-28], but to obtain predictable effects the stoichiometry between the different folding factors should be considered. Achieving the correct balance of folding factor expression is a laborious task and high-throughput techniques need to be implemented to strain development in order to gain improvements in protein production.

In this study, we demonstrate how yeast protein secretion can be enhanced by increasing the ER space through deletion of the OPI1-gene. By using this strain along with the wild-type, we conducted a comprehensive screening of six yeast folding factors under control of up to five different promoters to identify elements and expression levels that were beneficial for IgG secretion. Overexpression was combined with the expanded ER to produce a strain with high secretion levels. Our focus was on modifying the ER environment, and we discuss how the different modifications might affect heterologous protein production in yeast.

\section{Results and discussion}

\section{Increasing the size of yeast ER increases IgG secretion} levels by more than fourfold

The $S$. cerevisiae gene OPI1 is involved in regulation of lipid-metabolism, and its main target is the Ino2p/ Ino4p-complex. Ino2p and Ino4p are transcription factors that work as a heterodimer to induce the transcription of many phospholipid synthesis enzymes [29, 30]. The protein product of OPI1 functions as a repressor for the Ino2p/Ino4p-complex as Opi1p binds to Ino2p and inhibits the formation of the active heterodimer in lipid abundant conditions [31]. Removal of Opi1p by gene knock-out resulted in a constitutively active Ino2p/ Ino4p-complex, which upregulated the de novo synthesis of lipids $[15,29,30]$. The Ino2p/Ino4p-complex has a key role in ER size control, and removing its repressor Opilp causes the yeast ER to expand by approximately 1.5 -fold compared to the wild-type yeast ER [15].

Increase in ER size is a characteristic feature of professional secretory cell development [32, 33] and has been stated to reduce ER stress in S. cerevisiae [15], thus we decided to study whether increasing ER size before induction of protein production would correlate with an increased IgG yield in our system. We generated a yeast strain that, in addition to having a galactose-inducible cassette for encoding full-length human IgG inserted to its genome, had the OPI1-gene deleted. This strain, named here as $\triangle$ opil, was used throughout the screening along with the parental W $303 \alpha$-strain that had only the IgG expression cassette insertion, designated here as the wild-type (wt).

Growth of wt and $\Delta o p i 1$ strains was compared under non-inducing and inducing conditions for $40 \mathrm{~h}$ in complex YPD and synthetic defined media. Under non-inducing conditions, the $\Delta o p i 1$-mutant did not display any defects in growth, but reached a slightly lower optical cell 
density $\left(\mathrm{OD}_{600}\right)$ in complex media compared to the wildtype, in stationary phase the $\mathrm{OD}_{600}$ being 1.762 for wt and 1.698 for $\Delta$ opil yeast strain $(P$ value $<0.001)$ (Fig. 1a). The same behavior was recorded in SD media with raffinose as the carbon source (Fig. 1b). However, during IgG expression, the maximal specific growth rate $\left(\mu_{\max }\right)$ was diminished and the $\mu_{\max }$ was $0.110 \mathrm{~h}^{-1}$ for wt and $0.065 \mathrm{~h}^{-1}$ for $\Delta$ opi1 cells in YPGal (Fig. 1a). The effect on growth rate was not as severe in SGal medium (Fig. 1b). In SGal the maximum growth rates were $0.280 \mathrm{~h}^{-1}$ for wt and $0.266 \mathrm{~h}^{-1}$ for $\Delta$ opil. However, in the production conditions represented in Fig. 1b, the lag-time of $\Delta$ opi1 was prolonged. Despite generally lower $\mathrm{OD}_{600}$ values in the cultures, the mean antibody titer of $\Delta$ opi1 strain was up to three fold higher than that of the wild-type strain in the same conditions (Fig. 1c, Additional file 1). When the antibody titer was normalized to cell density, $\Delta$ opi1 performed up to 4.8-fold better than the wild-type in IgG-secretion when grown at $30{ }^{\circ} \mathrm{C}$ (Table 1, Additional file 1), clearly indicating that the characteristic increase in ER size by OPI1 deletion is beneficial for IgG production. A similar positive effect of ER size manipulation has been reported for membrane protein production where the rerouting of metabolic fluxes from storage lipids to phospholipid biosynthesis led to an increase in ER membrane and was accompanied by an improvement of membrane protein accumulation due to the increased folding space [34]. In addition to increasing the ER functional space and affecting UPR-response, deletion of the OPI1gene might also be beneficial for protein production
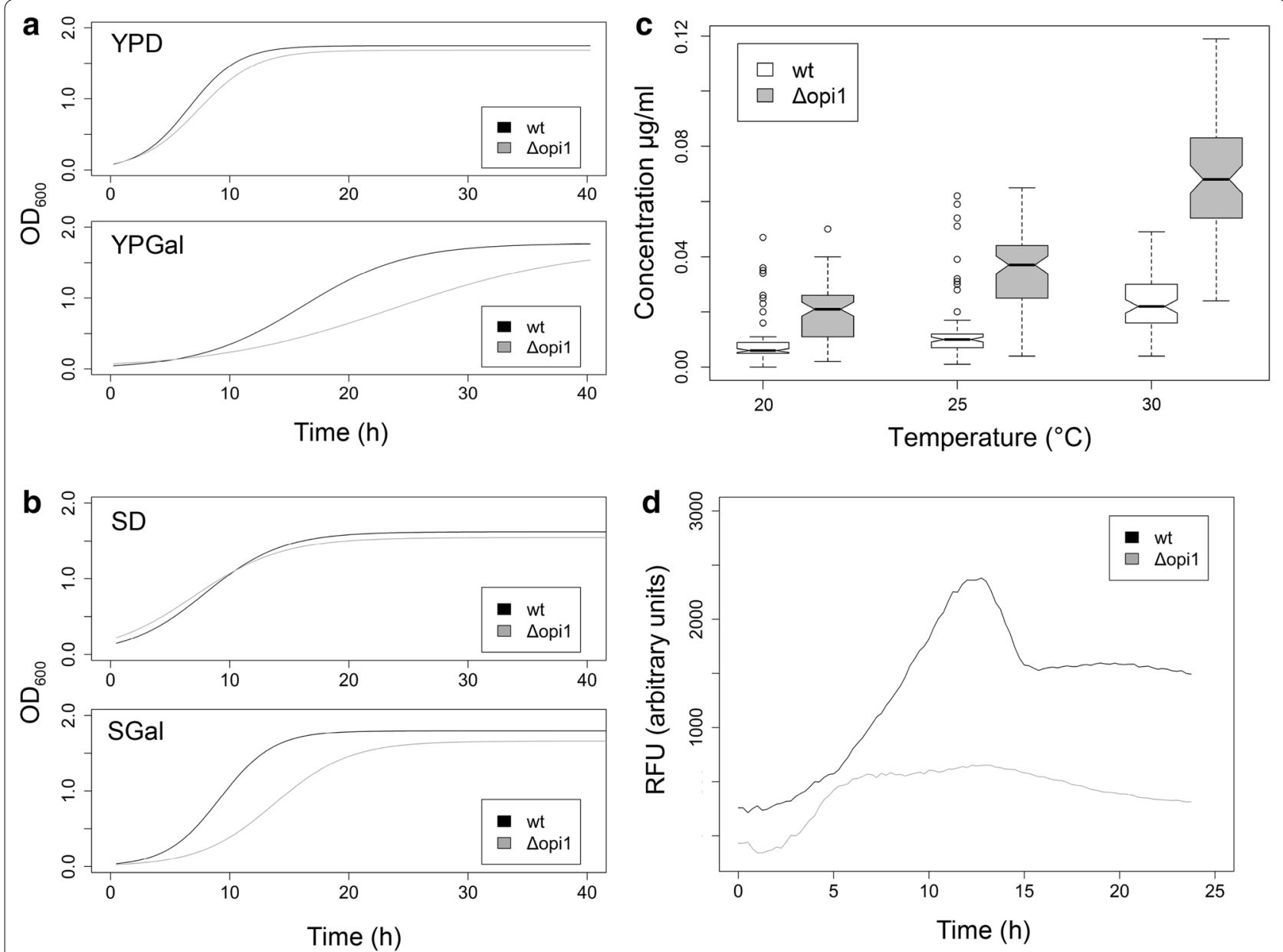

Fig. 1 Dopil deletion increases antibody production through increased ER size. a Growth of the two background strains was measured in complex (a) and synthetic media $(\mathbf{b})$ at $30^{\circ} \mathrm{C}$. Used media are indicated in the graphs. Upper panels represent growth under non-inducing conditions, lower panels growth under inducing conditions. When antibody expression was not induced (a and $\mathbf{b}$, upper panels), growth behavior of wild-type (wt) and $\triangle$ opil strains was similar. While antibody expression was induced (a and $\mathbf{b}$, lower panels), growth of $\triangle$ opil mutant was reduced. $\mathbf{c} A$ notched box-plot presentation of all the measured antibody concentrations for the background strains shows that antibody secretion was considerably more efficient for $\triangle$ opi1 in all temperatures. The number of measurement points used for each box is 82 or 84 . $\mathbf{d}$ Fluorescence of GFP under an UPRresponsive promoter was recorded in wt and $\triangle$ opi1. The background corrected RFU-values remain much lower for $\triangle$ opi1. Highest GFP fluorescence of wt was two times higher per OD compared to $\triangle$ opil 
Table 1 List of strains with the highest specific antibody product yields

\begin{tabular}{|c|c|c|c|c|c|c|}
\hline $\begin{array}{l}\text { Strain } \\
\text { number }\end{array}$ & Added elements & Strain & $\begin{array}{l}\text { Specific product yield } \\
\left(\mu \mathrm{ml}^{-1} \mathrm{OD} 600^{-1}\right)\end{array}$ & $\begin{array}{l}\text { Relative } \\
\text { frequency }^{a}\end{array}$ & P value & $\begin{array}{l}\text { Fold-change } \\
\text { relative to wild-type }\end{array}$ \\
\hline 154 & $C P R 5-P_{G P D}$ & $\triangle o p i 1$ & 0.2027 & 0.2 & 0.000002 & 10.22 \\
\hline 157 & $C P R 5-P_{K A R 2}$ & $\triangle o p i 1$ & 0.1992 & 0.15 & 0.000159 & 10.15 \\
\hline 155 & $C P R 5-P_{G A L 1}$ & $\triangle o p i 1$ & 0.1579 & 0.1 & 0.000560 & 7.99 \\
\hline 153 & $C P R 5-P_{\text {TEF }}$ & \opi1 & 0.1525 & 0.075 & 0.000036 & 7.66 \\
\hline 156 & $C P R 5-P_{P D / 1}$ & $\triangle o p i 1$ & 0.1424 & 0.05 & 0.007624 & 7.15 \\
\hline 170 & $E R O 1-P_{G A L 1}$ & Sopil & 0.1226 & 0.05 & 0.020502 & 6.13 \\
\hline 212 & $S I L 1-P_{G A L 1}$ & 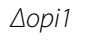 & 0.1223 & 0.05 & 0.021128 & 5.53 \\
\hline 162 & $P D / 1-P_{\text {TEF }}$ & $\Delta$ opi1 & 0.1208 & 0.075 & 0.279237 & 6.09 \\
\hline 206 & $L H S 1-P_{G P D}$ & \opi1 & 0.1163 & 0.05 & 0.299870 & 5.88 \\
\hline 402 & LHS1-P $P_{\text {KAR2 }}+S I L 1-P_{\text {KAR2 }}$ & \opi1 & 0.1139 & 0 & 0.011316 & 5.73 \\
\hline 83 & None & \opi1 & 0.0886 & 0.075 & & 4.47 \\
\hline 73 & None & wt & 0.0199 & 0 & & 1 \\
\hline
\end{tabular}

The data represent the mean value of the experiments conducted with 0.5 and $2 \%$ galactose induction at $30^{\circ} \mathrm{C}$

a Relative frequency in the 95th percentile of $\Delta$ opi1-strains

b Calculated with the nonparametric equivalent of $\mathrm{t}$ test, Wilcoxon signed rank test using the $\Delta$ opi 1 -strain without added elements as the reference

c Averages of the fold-changes relative to wild-type

because of the possible roles of its downstream effectors Ino4p and Ino2p in protein biosynthesis and transport [29], implying that Opi1p could also influence the protein secretion pathway through other, more complex effects.

Opilp inactivation was shown to have a direct effect on alleviating conformational stress [15], so we investigated the magnitude of the unfolded protein response under antibody expressing conditions in $\Delta o p i 1$ and wt (Fig. 1d). We integrated an UPR-responsive cassette to both strains, which expresses an intracellular GFPsignal upon induction of UPR [35]. Interestingly, in the beginning the increase of signal from the UPR-reporter occurred at the same rate in wt and $\Delta o p i 1$, but in the latter, signal increase persisted only during short time frame (Fig. 1d). As we used an intracellular reporter, growth might have an effect to the results. Nevertheless, if $\mathrm{OD}_{600}$ values are taken into account, during the height of UPRinduction levels in wt, the strain showed a much higher UPR-GFP signal than in $\triangle$ opi1 (Fig. $1 \mathrm{~d}$, around $13 \mathrm{~h}$ ). It is possible that the initial UPR-induction occurs at the same strength in wt and $\Delta o p i 1$, but the stress caused by the unfolded proteins is resolved faster in cells with a pre-enlarged ER. The UPR-signal persisted in wt until stationary phase of growth was reached, after which the reporter signal faded to a stationary level (Fig. 1d).

\section{High-throughput screening set-up for optimizing lgG production in yeast}

Adjusting the host organism for protein production is a complex process that requires optimization at several levels. The effects of single gene additions are hard to predict and are highly dependent on the client protein
[5, 6]. To improve S. cerevisiae as an IgG factory, we set up a screening scheme summarized in Fig. 2. We created a plasmid library of six factors that participate in protein folding, and to fine-tune their expression levels, we selected five promoters of different strength to diversify our expression library. The promoters included the constitutive promoters $\mathrm{P}_{\mathrm{GPD}}$ and $\mathrm{P}_{\mathrm{TEF}}$, the galactoseinducible GAL1-promoter $\mathrm{P}_{\mathrm{GAL} 1}$, which activates the expression of the folding factor along with the induction of client protein expression, and also two UPR-inducible promoters from yeast genes KAR2 and PDI1, $\mathrm{P}_{\mathrm{KAR} 2}$ and $\mathrm{P}_{\mathrm{PDI} 1}$ respectively, which differ in their basal and inducible expression levels [36]. Each promoter-gene combination was tested both in wild-type and $\Delta$ opi1 strains expressing human IgG. Based on the performances of the strains transformed with a single plasmid of the expression library, plasmids encoding proteins of different functions were combined for another round of testing. In total, we tested 52 individual gene-promoter-strain combinations, and 67 strains with two different gene-containing plasmids.

For initial testing of single elements, plasmids encoding the target genes were cotransformed with an empty plasmid with the complementary marker. This allowed us to use exactly the same media for initial testing and testing of combinations, as every strain contained both URA3 and LEU2 auxotrophic markers. The two background strain were transformed with two empty plasmids. In addition, this approach avoided bias in growth and metabolism, as different plasmid complementation has been shown to have an effect on various cellular processes [37]. All screening experiments were conducted 


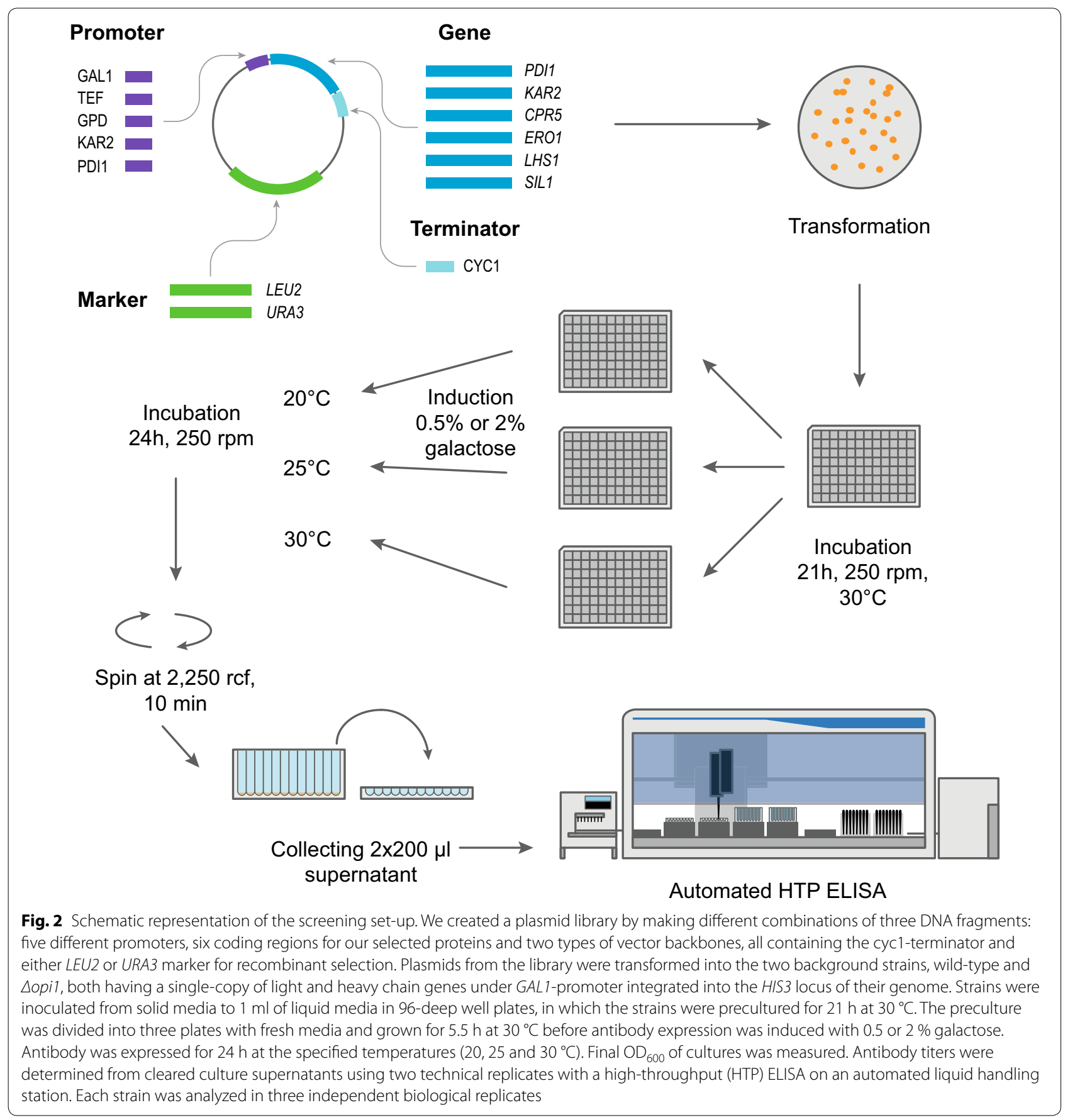

entirely in 96-deep well format, inoculating a colony from solid media to one $\mathrm{ml}$ of liquid media in each well. As shown in Fig. 2, after the preculture the cells were divided into three replicate plates. Replica plates were grown at 20,25 and $30^{\circ} \mathrm{C}$. For induction of IgG expression, galactose was added to final concentrations of 0.5 and $2 \%$. Each strain was cultivated three times, with inoculation of a different transformant and variation of the position in the culture plate to minimize plate position bias. The multiplexed conditions allowed us on one hand to gather better understanding about the link between a particular strain and antibody production and on the other hand on the effect of culture condition on IgG production. For analysis, $\mathrm{OD}_{600}$ was measured, and clarified supernatants were collected in duplicates from deep-well plates to determine the antibody titer with automated ELISA. 


\section{Antibody secretion increased with temperature and the effect of OPI1-gene deletion was preserved in all transformant strains}

Throughout all conditions screened, the two genetic backgrounds manifested as two distinguishable populations, as evidenced by differences in antibody production (Fig. 3a), and growth (Fig. 3b). The corresponding distributions of specific product yields of the biological replicates are represented in Fig. 3c and individual replicates expand up to $0.3 \mu \mathrm{g} \mathrm{ml} \mathrm{m}^{-1} \mathrm{OD}_{600}^{-1}$. The antibody production increased with temperature (Fig. 3a) along with the OD-values (Fig. 3b), as specific product yield increased to a lesser extent (Fig. 3c). The $\Delta$ opil strains reached generally higher antibody titers and lower $\mathrm{OD}_{600}$ values manifesting in higher specific product yields, as also was shown for the background strains in Fig. 1. In the $\Delta$ opil strains the effects of the added elements were mainly similar as in wild-type, but the relative changes in specific product yield were generally smaller when compared to the wt strain background (Additional files $1,2,3)$. It has been reported that ER expansion in $\Delta$ opi1 strain does not affect the intrinsic cellular levels of ER chaperones, so the functional capacity of ER, defined as the amounts of chaperones and other folding assistants, would not increase according to the size [15]. This might indicate that a diluted ER environment is less prone for IgG aggregation and thus less sensitive to the effects of chaperone overexpression. On the other hand, the clear improvement in antibody secretion achieved with OPI1gene deletion was preserved also during chaperone overexpression (Fig. 3c; Additional files 1, 3).

In total, we identified 49 cases where a more than $50 \%$ increase in antibody titers was measured and 71 cases where a more than 1.5-fold increase of the specific product yield was recorded when compared to the respective strain background. One question that arose from these experiments was whether the higher antibody titers were due to the increased growth or to increased specific product yield. To estimate this, we fitted a linear model to all data points (Fig. 3d). The mean values of $\mathrm{OD}_{600}$ and antibody titer from each strain were normalized to the respective background strain values to fit all the conditions to the same value space. A linear regression plane was fitted to the data points to model the dependency of the normalized antibody titer on the normalized specific product yield and normalized $\mathrm{OD}_{600}$. The multiple R-squared value for the model was 0.8896 , indicating that there was a significant linear dependency. The model estimated the coefficients to be 0.6114 for $\mathrm{OD}_{600}$ and 0.8573 for the specific product yield, indicating that improved specific product yield has a bigger positive effect on antibody titer than an increase in $\mathrm{OD}_{600}$. The linear model implies that the effects of the elements on secreted antibody amounts are more dependent on changes in cellular productivity than on effects in growth. The calculated $P$ values for the model fit measured by $F$ test and also for the coefficients are $\ll 0.001$ representing a reliable statistical significance for the linear model.

Both $\mathrm{OD}_{600}$ and antibody titer values increased steadily with the temperature (Fig. 3a, b), and the absolute differences between different strains were most prominent at $30{ }^{\circ} \mathrm{C}$. The differences manifested themselves by a wider range of antibody titers at $30{ }^{\circ} \mathrm{C}$ (Fig. 3a) and the increasing variance in the strain antibody titer indicates (Additional files 1,3), that the strain phenotypes diverge more with increasing temperature and thus are more clearly visible at $30{ }^{\circ} \mathrm{C}$. The induction strength also had effect on the IgG secretion, mainly secreting higher amounts at $0.5 \%$ galactose (at $30{ }^{\circ} \mathrm{C}$, mean antibody concentration was $0.0254 \mathrm{gg} \mathrm{ml}^{-1}$ for $0.5 \%$ galactose and $0.0207 \mu \mathrm{g} \mathrm{ml}^{-1}$ for $2 \%$ galactose for all strains with wild-type background). When antibody concentration was normalized to $\mathrm{OD}_{600}$, the specific product yield did not differ significantly for the two induction conditions (respective values are 0.0210 and $0.0201 \mu \mathrm{g} \mathrm{ml}^{-1} \mathrm{OD}_{600}^{-1}$ for 0.5 and $2.0 \%$ galactose, respectively). Since the two induction strengths resulted in similar outcomes, we focus on $0.5 \%$ galactose in our analysis as it yielded higher concentrations of secreted IgG. The results from all strains tested along with the two background strains are reported in Additional files 1, 3.

(See figure on next page.)

Fig. 3 Population analysis of total screening data. Even with added genes, the two background strains perform differently as determined with antibody titer (a) and optical density $(\mathbf{b})$, forming two populations of measurement values. $\Delta$ opi1 (dashed red line) had generally higher antibody concentrations (a) and lower $\mathrm{OD}_{600}$-values (b) than the wild-type (black solid line) at all temperatures tested. Distribution of the specific product yields calculated separately for each sample and replicate are shown in (c). The distribution of values is shown as density plots, where the area under the line is equal to 1 (total probability of a measurement value being under the line) and the $y$-axis is proportional to relative frequency. The range of the measured values is given in the $x$-axis. Each condition included the analysis of 113 different strains and the number of measurement points in each curve is between 798 and 824 in (a) and 399-412 in (b) and (c). The mean values for each strain were normalized to the respective background strain and plotted together to represent all the measured values (d). A linear regression plane (dashed lines) was fitted to the data points to model the dependency of antibody titer on combined effects of $\mathrm{OD}_{600}$ and normalized specific product yield. Multiple R-squared was equal to 0.8896 indicating that a linear dependency was found. The estimated coefficient of fold-change was higher than that for the normalized $\mathrm{OD}_{600}$, meaning that a change in specific product yield had a higher impact on the antibody titer values. Colors are added only to aid the visual clarity in (d) 

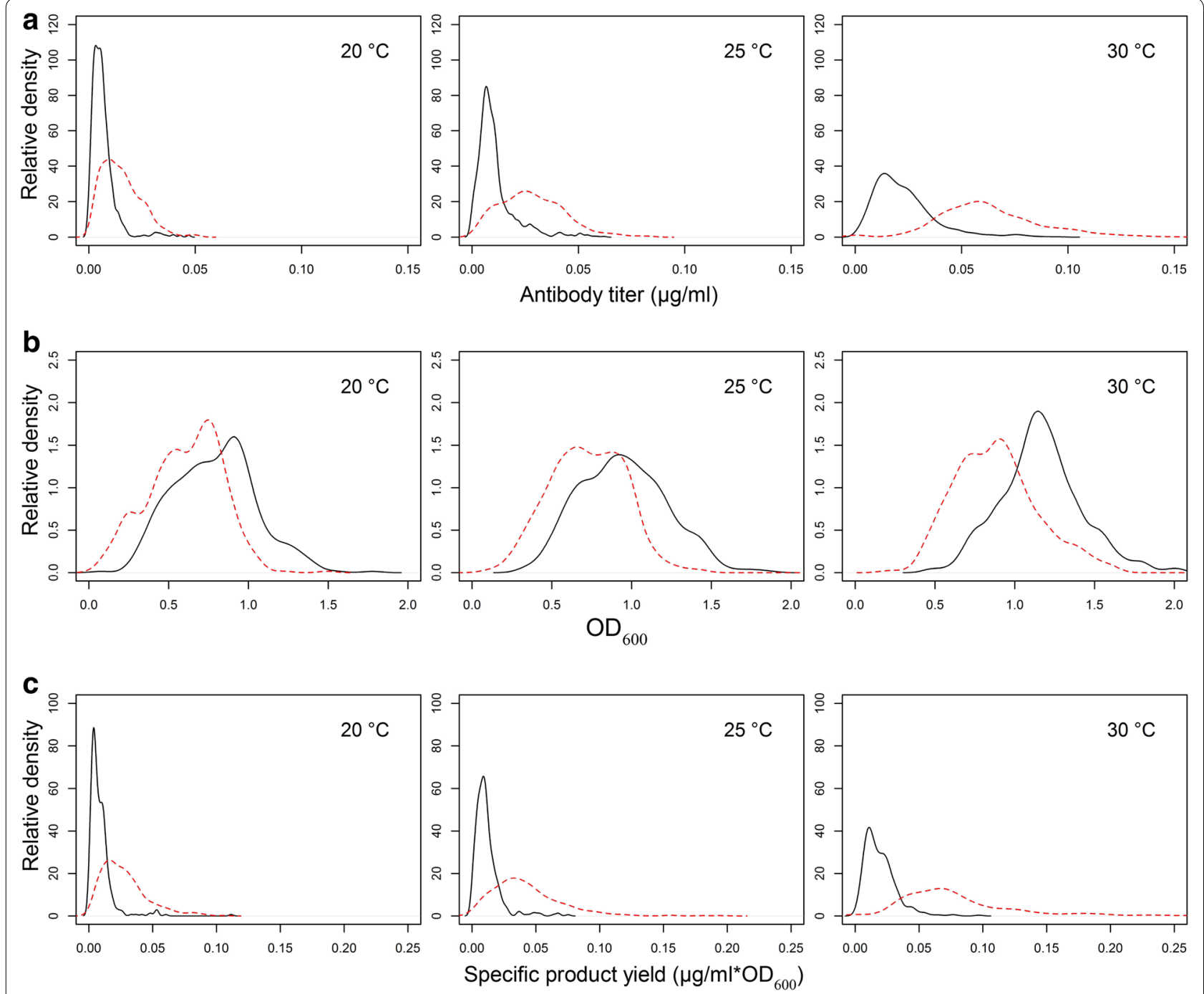

d

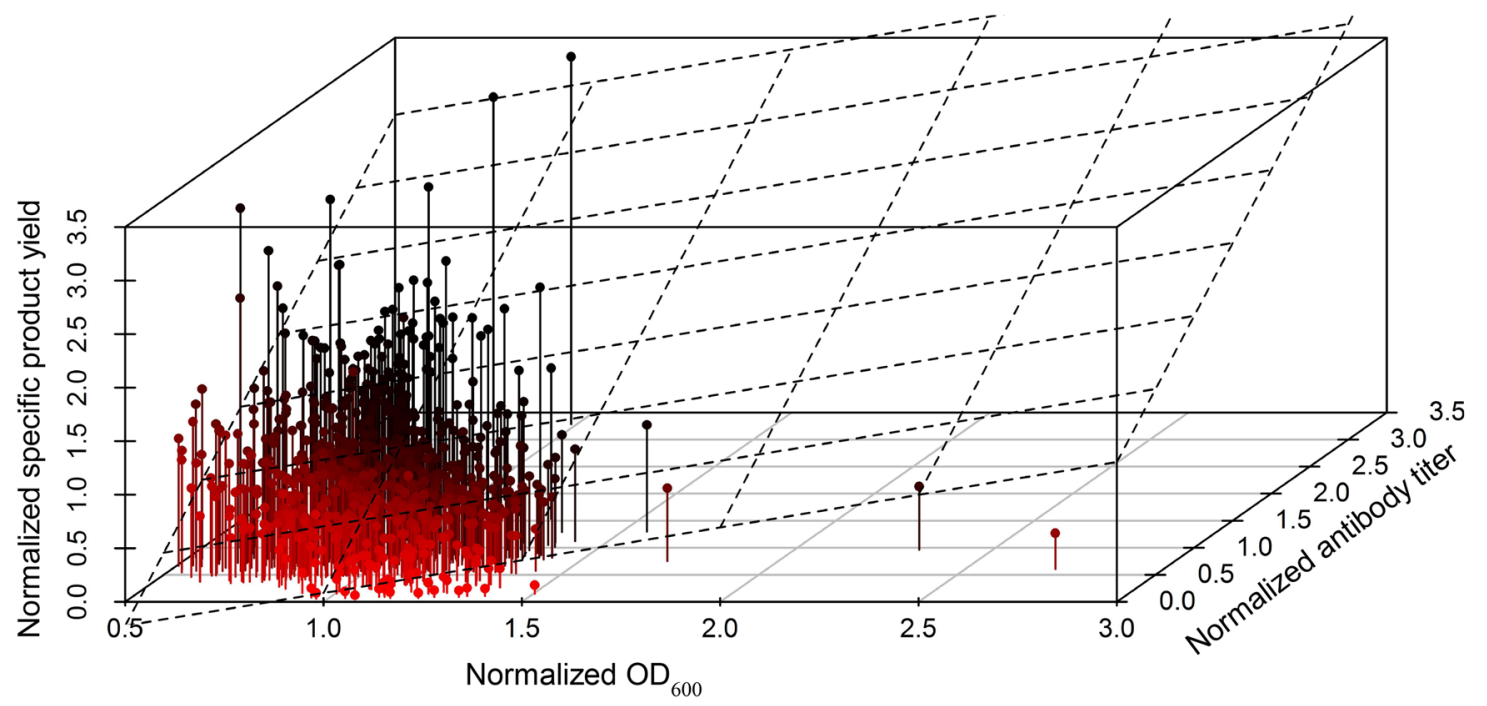




\section{The peptidyl-prolyl isomerase CPR5, a novel player} to increase antibody production

Antibody titers/specific product yields varied according to chaperone or helper protein, but also according to the promoter used. Figure 4a presents a heatmap that displays a summary of the fold changes in specific product yield that the added elements induced in the wildtype strain and Fig. $4 \mathrm{~b}$ is a similar heatmap for the $\Delta$ opi 1 strain, both at $30{ }^{\circ} \mathrm{C}$ with $0.5 \%$ galactose induction. For comparison, heatmaps from $2 \%$ galactose induction are included in Additional file 2, displaying that in general, the effects of the elements are similar in different strain backgrounds and conditions.

Standing out as the most beneficial gene for increasing antibody titers, CPR5 expression improved the specific product yield 1.65-3.25-fold depending on the promoter and condition used. Cpr5p is an ER localized PPIase involved in the rearrangement of peptidyl-prolyl
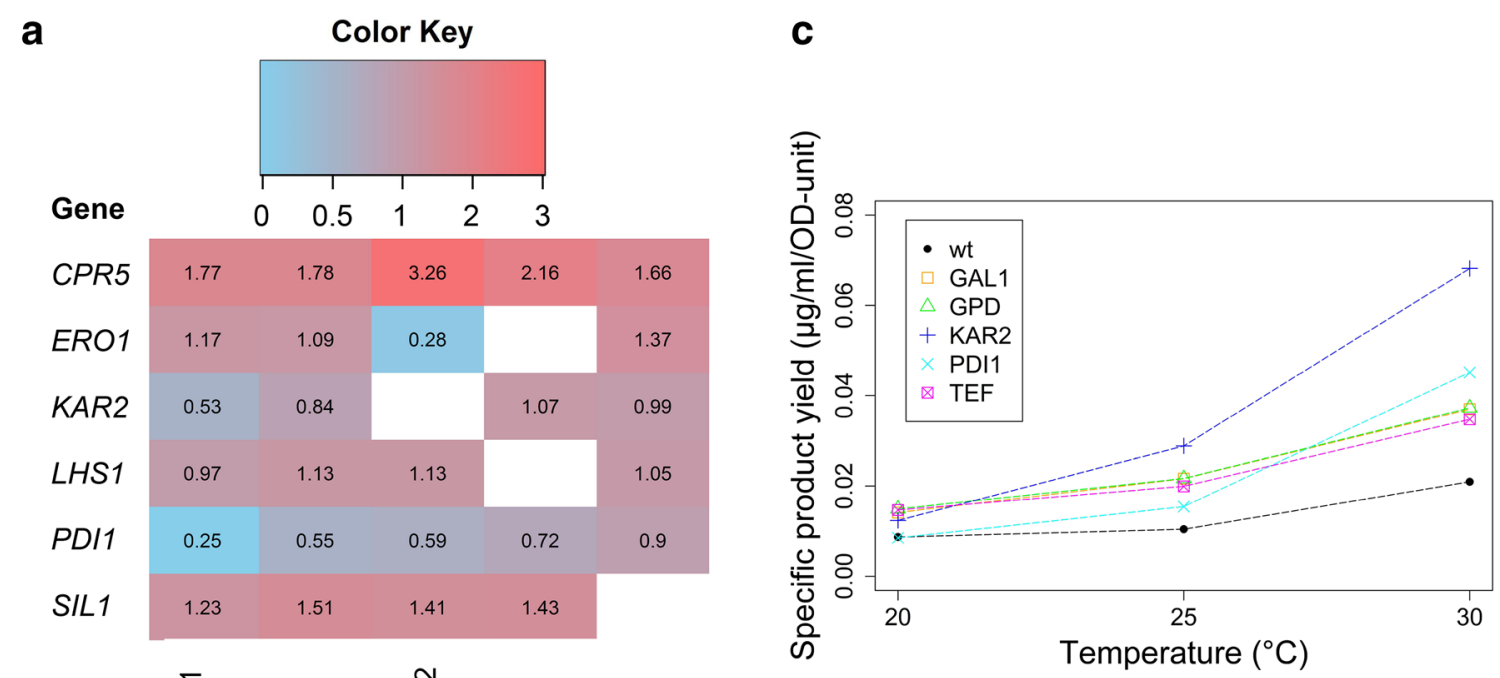

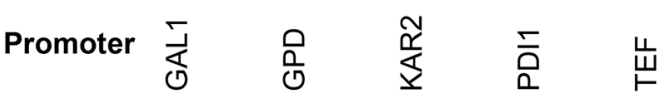

b

Gene

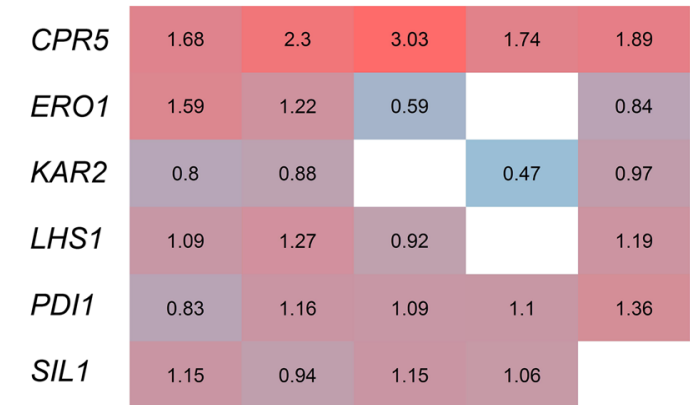

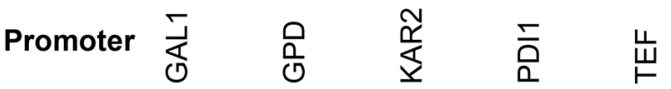

d

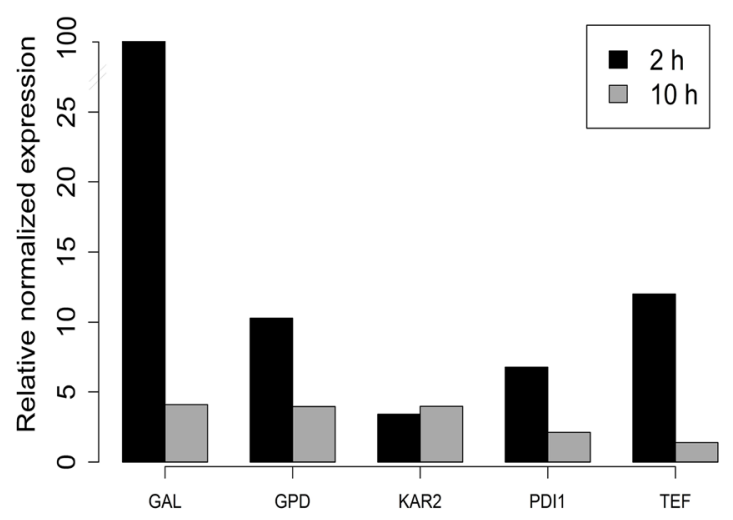

Fig. 4 Effects of single folding factor overexpression on antibody secretion. A heatmap representing the change in specific product yield of each strain relative to the strain background. The colors and numbers in each cells represent the fold-change that the specific promoter gene combination had on the specific product yield. CPR5 overexpression yielded the most beneficial impact. The values are for wild-type yeast strains grown at $30^{\circ} \mathrm{C}$ and for $0.5 \%$ galactose induction (a) and $\triangle$ opil strains in the same conditions $(\mathbf{b})$. c Temperature and type of promoter affect the effect of CPR5 on the specific product yield in wild-type. UPR-controlled promoters, $P_{K A R 2}$ and $P_{\text {PDI1, }}$ show an increase in the positive impact on antibody secretion with increasing temperature, as displayed by the trend line. The specific product yield of wild-type is included as comparison. The values are from measurements with $0.5 \%$ galactose induction. d Promoter strengths measured by qPCR of CPR5 mRNA levels at 2 and $10 \mathrm{~h}$ after induction with $2 \%$ galactose at $30^{\circ} \mathrm{C}$. Relative normalized expression is calculated as setting the CPR5 mRNA levels from wt as control. The promoters show different characteristics, as the UPR-inducible promoters follow the wt levels and $\mathrm{P}_{\mathrm{GAL} 1}, \mathrm{P}_{\mathrm{GPD}}$ and $\mathrm{P}_{\mathrm{TEF}}$ have higher initial mRNA amount 
bonds in unfolded proteins [38]. PPIases have often been neglected in studies of recombinant protein folding and production, although specifically with full-length antibodies, the isomerization of certain proline residues has been determined to be the rate-limiting step in antibody folding in some cases [21, 39-41]. Feige et al. reported also that immunoglobulin domain folding in vitro could be enhanced by addition of the cytoplasmic yeast PPIase Cpr6p [41]. In our study, the best results of CPR5 expression were achieved with the UPR-induced promoter $\mathrm{P}_{\mathrm{KAR} 2}$, with which the strains showed to be secreting the most IgG at $30{ }^{\circ} \mathrm{C}$ (Fig. 4b). As seen in Fig. 4c, the effects of the individual elements were not equal at the different temperatures, as also can be stated for other elements, but generally the induced changes in specific product yield were to the same direction (Additional files 1, 3).

The nucleotide exchange factors Sil1p and Lhs1p are regulating Kar2p activity by maintaining its ATPase cycle through ATP hydrolysis and subsequent ADP release [42, 43]. In Fig. 4a, b we show that using the Kar2p co-chaperones was successful in improving antibody titers, Sillp showed a clear positive effect (up to 1.5-fold higher secretion), especially in wt background (Fig. 4a), and Lhs1p induced a modest increase in titers. These co-chaperones have been used before to improve the overexpression of human transferrin and recombinant human albumin, although with slightly lower fold-changes [44].

Although overexpression of Pdi1p and Kar2p have been shown to improve the secretion of single-chain antibodies in S. cerevisiae [26-28], in our analysis both of these proteins displayed mostly negative effects on antibody secretion at all induction levels and expression conditions (Fig. 4, Additional files 1,2). The considerable reduction in IgG secretion when overexpressing PDI1 under strong promoters $\left(\mathrm{P}_{\mathrm{GAL}}, \mathrm{P}_{\mathrm{GPD}}\right)$ was rather surprising, as its overexpression has shown to be increasing titers for various heterologous proteins [45-47]. Besides a possible disruption of the cellular redox balance by a too high concentration of Pdilp [48], there has also been evidence that the protein facilitates the ERAD of some substrates [49]. The importance of oxidative balance can be also seen from the effects of Erolp, another redox-active protein, as the secretion of IgG shows to vary greatly depending on the expression level of ERO1 (Fig. 4a, b; Additional file 2).

Under normal conditions, Kar2p associates with Ire1p in the ER and so represses UPR-induction. When unfolded proteins are present, Kar2p releases from Ire1p and binds them, after that Ire1p can dimerize and induce the UPR $[8,50]$. It has been shown that overexpression of Kar2p attenuates Ire1p activation [51] and this makes it possible that the overexpression leads to decreased IgG secretion through an inhibition of the UPR and thus accumulation of the unfolded IgG in the ER. Our data above showed that it is more reasonable to concentrate on regulating the activity of Kar2p by the expression of its co-chaperones Sil1p and Lhs1p, as improved Kar2p cycling might benefit the folding process. It has been estimated that Kar2p is by far the most abundant protein of this trio, with around 300,000 molecules per cell, whereas of Sillp and Lhs1p around 2400 and 140 molecules are present in the ER [52]. This indicates that increasing the abundance of the regulators can have great effects on Kar2p function. For the production of IgG, this is further supported by data that the Kar2p mammalian homolog BiP does not cycle away efficiently from both unassembled heavy and light chain $[53,54]$, which might stall protein folding at high Kar2p levels in the ER.

To estimate the extent of chaperone overexpression, we measured the mRNA levels of CPR5 with qPCR from wt strains overexpressing CPR5 under control of different promoters 2 and $10 \mathrm{~h}$ after induction (Fig. 4d). Compared to the wt strain harboring only the native gene copy under control of the endogenous promoter, CPR5 expression under control of GAL1, GPD and TEF promoters resulted in a very high initial induction of CPR5 expression, especially under GAL1-promoter (Fig. 4d). Ten hours after induction, CPR5 mRNA abundance was comparable in all strains harboring an overexpression plasmid. Interestingly, expression of CPR5 under control of $\mathrm{P}_{P D I 1}$ or $\mathrm{P}_{K A R 2}$ was only slightly enhanced at both time points compared to the control (Fig. 4d). The data indicates that using UPR-responsive promoters enables a moderate overexpression that stays more consistent throughout the production process. The UPR-induced promoters proved to be promising and should be considered when chaperones are overexpressed to enhance heterologous protein production.

\section{Combining different genes provided little additional benefit to IgG production}

After evaluating all the individual elements and determining the best promoter for each gene, we started screening for gene combinations that would further improve IgG secretion. For selecting the combinations, a rational approach was applied based on the known functionalities and interactions of the genes aiming to achieve complementary actions. Optimal expression levels of the gene pairs are crucial but most likely hard to predict, so for the selected pairs we tested a few promoter options. All the selected pairs along with all the measurement results from the screening process are listed in detail in Additional file 3, while Additional file 4 provides a summarized overview of the mean fold-changes. The measurements from the second round are included in Fig. 3, thus the general trends discussed above apply to double 
gene combinations as well. Again we focus mainly on the results of the experiments performed at $30^{\circ} \mathrm{C}$, as in these conditions the highest antibody titers were obtained and thus the conditions are most interesting for further applications.

We did not discover any gene pairs with beneficial synergistic or combinatorial effects. When improving, the fold-changes in overexpression of two elements were less than achieved with just one element. For example, CPR5 expressed alone induced a significant increase in IgG secretion, but combining the overexpression with other elements, for example LHS1 or SIL1, resulted in no more than 2.3 -fold increase at $30^{\circ} \mathrm{C}$ (biggest fold-change with CPR5-P $P_{K A R 2} / L H S 1-P_{K A R 2}$ combination, see Additional file 3), lower than the observed three-fold for CPR5 alone. As shown in Fig. 5, negative synergistic effects did also occur, often with Kar2p overexpression. The poor performance of two element combinations is reflected by their high occurrence in the lowest, 5 th percentile of measurement points (Additional file 5). Targeting of multiple proteins to the ER might be too demanding for the cell, resulting in protein production stress and high occupancy in the translation/translocation machinery thus leading to inefficient secretion. Also, with the overexpression of more folding factors, there is a higher chance to disturb other cellular processes, which might have an indirect combinatorial negative effect on antibody titers. However, overall we did not observe any clear changes in growth when compared to the strains that express only a single element, indicating again that the secretion levels are more dependent on the ability of cells to process IgG and not on changes in OD-values as described in Fig. 3d.

Co-overexpressing Lhs1p and Sillp, both under $\mathrm{P}_{\text {KAR2 }}$, increased the specific product yield by up to 1.5 -fold (Fig. 5, Additional files 3, 4). As Lhs1p and Sil1p control Kar2p activity by determining the rate of the ATPphosphorylation cycle [42], overexpressing both of them might be important to adjust the speed of the Kar2p binding cycle to the increased demand for substrate folding. Although LHS1- $\mathrm{P}_{K A R 2} / S I L 1-\mathrm{P}_{K A R 2}$ was one of the best combinations in enhancing the specific product yield, an effect of the same magnitude was achieved by overexpressing Sillp alone. This indicates that Sil1p is the main protein providing the ADP release step in Kar2p cycling. Interestingly, as with CPR5 overexpression, the higher antibody titers of the LHS1- $\mathrm{P}_{\text {KAR2 }} /$ SIL1- $\mathrm{P}_{\text {KAR2 }}$ were accompanied by a slightly lower OD-values (Additional files 3,4$)$. There might be significant competition for cellular resources between high level protein production and growth [55-57], which might also explain why addition of multiple folding factors did not produce higher antibody titers.

\section{The strain with highest specific product yield, $\triangle o p i 1+C P R 5-P_{\mathrm{GPD}}$, has 10-fold higher specific IgG yields than the wild-type}

Overexpression of Cpr5p under any promoter proved to yield the highest amounts of secreted IgG per cell. When combined with the $\Delta$ opi1-mutation, the specific product yield could be increased up to 10.2-fold compared
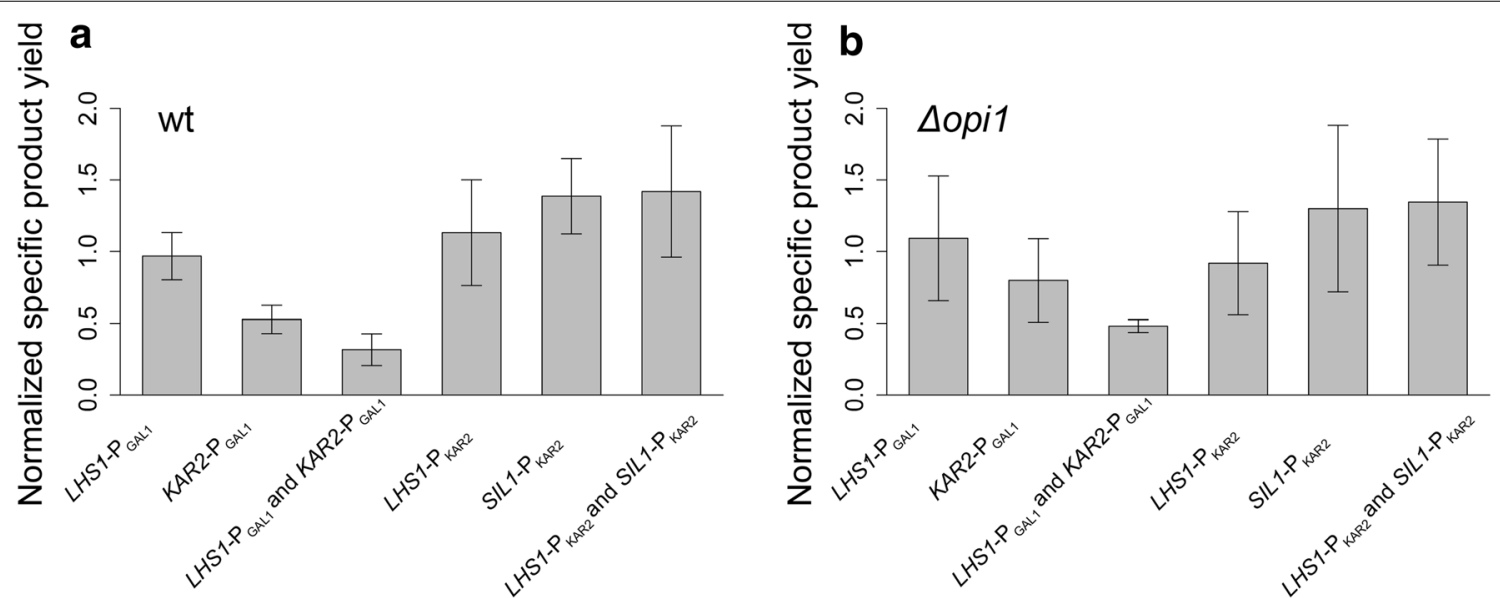

Fig. 5 Performance of two-gene combinations compared to the overexpression of only one of the genes. Bar plots showing the relative specific product yield for selected gene combinations and the respective single genes in wild-type (a) and $\triangle$ opi1 yeast strains (b). LHS1- $\mathrm{P}_{\text {GAL1 }}$ and KAR2$P_{\text {GAL1 }}$ seem to have synergistic negative effect since their combination decreases the specific product yield below the level of the combined or individual effects. LHS1-P KAR2 $_{\text {with SIL1-P }}$ KAR2 had the most positive effect on specific product yield out of all the double gene combinations, but the increase was at the same level as achieved by insertion of SIL1- $P_{\text {KAR2 }}$ alone. Although the effects of the added genes are similar for $\triangle$ opi1 (b), the effects are higher in magnitude in wild-type strain background (a). Error bars represent the standard deviation and the selected values were obtained with $0.5 \%$ galactose induction at $30^{\circ} \mathrm{C}$. Specific product yield is normalized to the mean specific product yield of the respective background strain grown in the same conditions 
to the wild-type in the same conditions. The ten strains with highest mean specific product yields are listed in Table 1. Based solely on $0.5 \%$ induction, as shown in Fig. 4b, CPR5-P $\mathrm{P}_{\text {KAR2 }}$ provided most enhancement with the Aopi1-strain. However, when evaluating a combination of the 0.5 and $2 \%$ conditions, overexpression of CPR5 under the GPD-promoter provided the highest mean improvement in specific product yield. The $\Delta o p i 1+C P R 5-\mathrm{P}_{\mathrm{GPD}}$ strain yielded specific antibody titers of around $200 \mathrm{ng} \mathrm{ml}^{-} 1 \mathrm{OD}_{600}^{-1}$, corresponding to a final antibody concentration of $126 \mu \mathrm{g} \mathrm{l}{ }^{-1}$ with $0.5 \%$ galactose induction which is five times more than with the wild-type strain (Table 1; Additional file 1). Although our yeast system was significantly improved, the final antibody concentrations we achieved remained very modest. Many mammalian systems and cell lines, such as Chinese hamster ovary (CHO), HEK293, and a new cell line called F2N78 [58-60], achieve titers in the scale of hundreds of milligrams per liter after unprecedented cell line selection and engineering efforts. Our titers are several magnitudes lower, but comparing different systems is not straightforward in terms of parameters such as final concentration. Performance of different platforms in recombinant protein production was studied by Maccani et al. [61], where the authors show that based on the spacetime yield of a single chain antibody, $\mathrm{CHO}$ cells perform only 10 times better than the yeast Pichia pastoris. Our approach of expressing full-length antibodies differs from the common use of single chain antibodies as the client protein, so it is expected for our variant to display lower yields due to increased complexity. A fivefold increase in space-time yield was obtained here by optimizing just one aspect (folding and ER environment). There are still many approaches left for improving strain performance and yields including many genetic targets and selection of a strain background more suitable for protein production. Together with process optimization, final antibody concentration could reach economically feasible levels in the future.

In Table 1, we display the mean values for the specific product yield at $30{ }^{\circ} \mathrm{C}$ for the two induction strengths, as combining these two conditions gives a more reliable estimation for the quantitative change for each element. Within some strains, we observed a high variance between measurements, especially at high antibody titers, probably resulting from a combined effect of differences in cellular state and unavoidable technical inaccuracy (high SD values in Additional files 1 and 4, also Fig. 5). To validate the results of the most different phenotypes, we conducted frequency analysis to determine which strains were enriched among the lowest (5th percentile) and highest (95th percentile) five percent of all measured specific product yields, divided by strain background and temperature (Additional file 5). Representing some outlier measurements, a few instances from the wild-type background strain occurred in both percentiles, since at least one replicate was grown on each deep-well plate to assess the overall success of the plate culture. Many of the same elements occur in the same percentiles in both background strains and different temperatures, indicating that the changes from the elements are generally consistent throughout the assay. Another measure of the reliability of the fold-change of the added elements is the low P-value (as determined by Wilcoxon signed rank test) calculated for many of the strains when compared to the respective strain background. Combination of low $\mathrm{P}$ value and high frequency displays a statistically significant fold change and very reliable results for the five best strains with overexpressed Cpr5p, and to most other distinctive phenotypes that we encountered (Table 1, Additional file 5).

\section{Analysis of cellular IgG clearance from selected strains}

When using cellular engineering to improve heterologous protein production, it is also useful to gain understanding of the underlying changes in strains with interesting secretion phenotypes. To gain more insight in how some of the overexpressed folding factors influence the progression of the antibody through the cell, we selected five strains for use in a cellular clearance assay as described in De Ruijter and Frey [12]. The strains were as follows: the two genetic strain backgrounds; wt and Sopi1, the highest producers from both backgrounds; wt with the CPR5- $\mathrm{P}_{\mathrm{KAR} 2}$ plasmid, and $\triangle o p i 1$ with the CPR5- $\mathrm{P}_{\mathrm{GPD}}$ plasmid, and finally one of the strains with a negatively affecting gene combination: $\Delta o p i 1$ with the KAR2- $\mathrm{P}_{\mathrm{GAL} 1}$ and the LHS1- $\mathrm{P}_{\mathrm{GAL} 1}$ plasmids. In short, cultures in the $\log$-phase were induced with $2 \%$ galactose for $4 \mathrm{~h}$, after which $2 \%$ glucose was added to inhibit expression from the GAL1 promoter. After 0, 2, 4 and $6 \mathrm{~h}$ cell extracts were prepared, which were analyzed for heavy $(\mathrm{HC})$ and light chain (LC) content using SDS-PAGE and Western blot. For quantification of IgG signals, the corresponding anti-tubulin signal was used to correct for differences in protein loading. The resulting Western blots are shown in Fig. 6a, c.

With the mock treatment, all strains kept producing IgG for the duration of the assay, as seen by a continuously present signal for the heavy and light chain blots (Control columns of Fig. 6a, c). When IgG expression was repressed by addition of glucose, intracellular IgG levels decreased during the assay and no $\mathrm{HC}$ was detected in the samples after $6 \mathrm{~h}$. However, most of the LC samples showed still residual signals at the last time point. Clearance curves were calculated for heavy and light chain that were corrected for loading with the quantified tubulin 

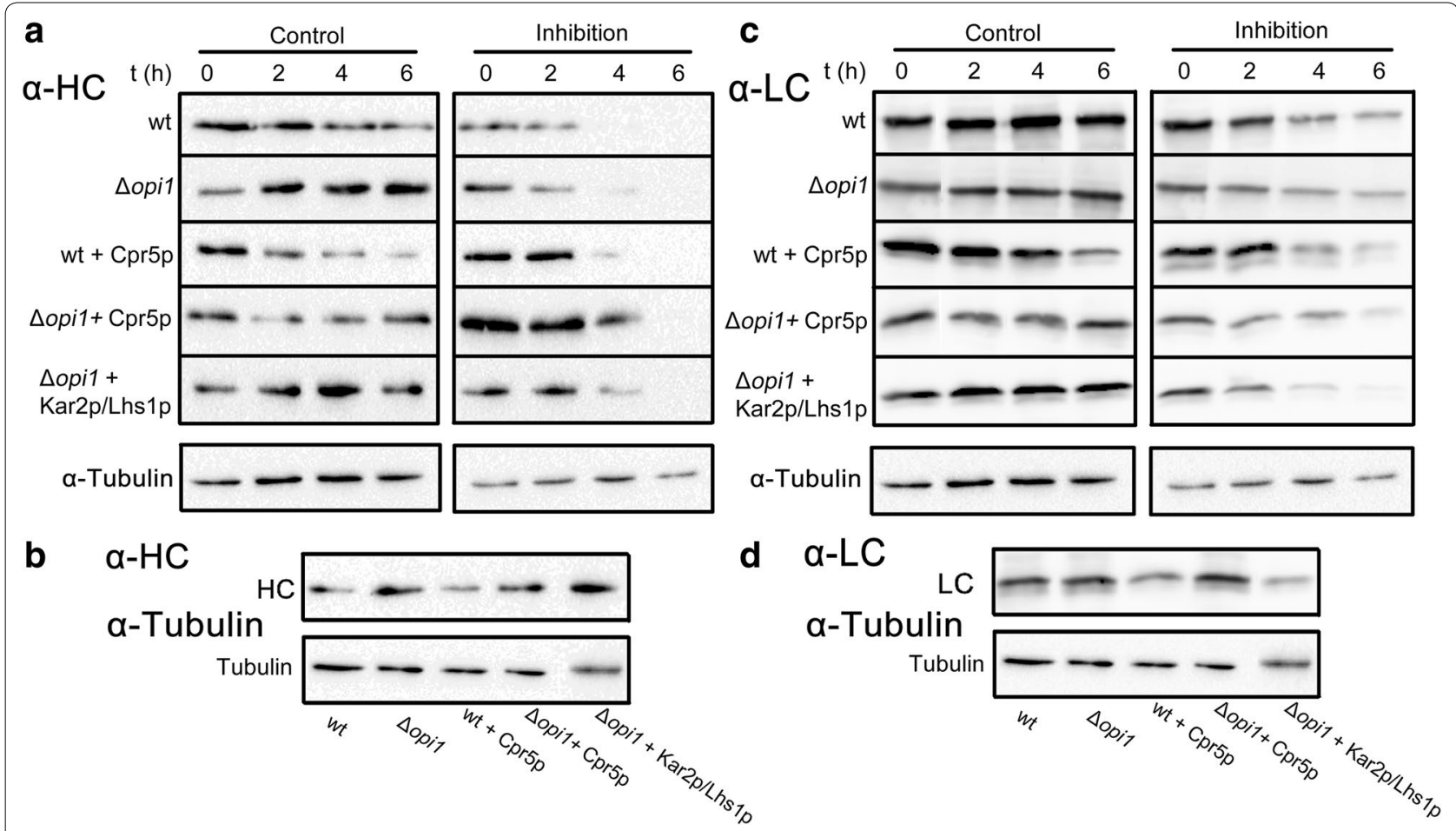

Fig. 6 Analysis of cellular lgG clearance using Western blot. a Antibody expression in exponentially growing cultures of different strains (wt, $\triangle 0 p i 1$,

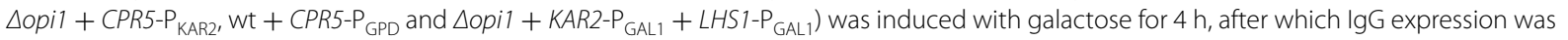
repressed by addition of glucose, in control experiments, the induction was continued. Heavy chain (HC) clearance was followed for 0, 2, 4 and $6 \mathrm{~h}$. After incubations, cell extracts were prepared from the cultures and analyzed using SDS-PAGE and Western blot. A representative blot for tubulin, used as loading control is shown. b Samples from time point zero from all strains were loaded on one gel, and the measured HC signals were visualized using Western blot, their respective tubulin signals are shown as loading controls. c Same as in (a), but samples were reprobed to visualize the light chain (LC) signals. $\mathbf{d}$ Same as in (b), but samples were reprobed to visualize the LC signals

signals from the glucose treated samples. The relative amount of signal was calculated with respect to the zero time point and the resulting clearance curves are shown in Fig. 7, which shows that HC and LC follow different clearance patterns from each other and between strains. This is not unexpected, as the light chain can be secreted separately, as opposed to the heavy chain that needs the light chain to correctly fold into the full IgG molecule in order to be secreted [21].

For a relative comparison of the intracellular load of $\mathrm{HC}$ and $\mathrm{LC}$ at the start of the glucose treatment, the samples from zero hours for each strains were compared for $\mathrm{HC}$ and LC signal intensities in Fig. 6b, d respectively. A notable difference was the increased intracellular $\mathrm{HC}$ concentration in the Sopil derived strains compared with the wild-type derived strains, which was accompanied with a slightly slower clearance. The $\Delta o p i 1$ strain had an around $75 \%$ higher initial HC load when compared with the wt strain and the best producing $\Delta$ opi1 overexpressing Cpr5p strain had more than two times higher HC load compared to wt Cpr5p overexpressing strain. Overall, a trend towards a higher intracellular HC load was detected in the $\Delta$ opi1 background. It has been shown for $\mathrm{CHO}$ cells that a high cellular content of $\mathrm{HC}$ is a molecular marker that characterizes efficient producing clones [62]. The same study showed also a lower correlation between LC content and productivity, while it has been shown that LC content has a strong correlation with strain productivity in heterohybridoma cells [63]. Although the intracellular LC levels in the analyzed best producing $\Delta o p i 1$ strains are quite similar to wt (Fig. 6d), the clearance of LC is slower in these strains (Fig. 7b), indicating that it might be favorable to have a pool of LC available for full IgG assembly.

The $\Delta o p i 1+$ KAR2- $\mathrm{P}_{\mathrm{GAL} 1}+$ LHS1- $\mathrm{P}_{\mathrm{GAL} 1}$ strain, which was showing a decreased IgG secretion, was included in this assay and showed a different pattern from the other strains. Even with a relatively low initial HC (after correction for loading) and LC load compared with the background $\Delta o p i 1$ strain, the clearance of $\mathrm{HC}$ was decreased and the clearance of LC was increased (Fig. 7a, b respectively). These results from our 

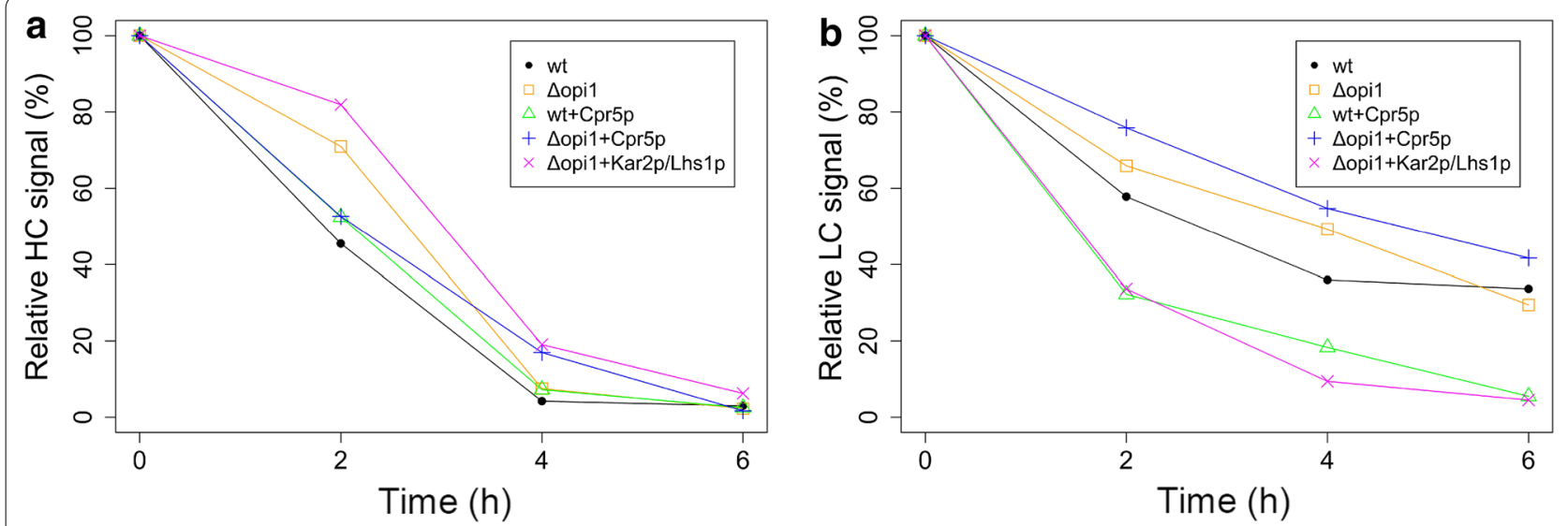

Fig. 7 Quantitative analysis of lgG clearance. Signals from the blots (HC and LC) in Fig. 6a and c were quantified and normalized with their corresponding tubulin signals and used to plot their clearance curves in (a) and (b), respectively. The data represents the average of two or three blots normalized for loading differences using an anti-tubulin blot. There is a clear variation between HC and LC clearance for all strains and also between the strains in both of the plots. The data gives a general indication that a constant pool of available LC might be beneficial for efficient IgG folding and secretion

clearance analysis indicate that a constant availability of $\mathrm{LC}$ in the cells might be beneficial for an efficient secretion of fully folded IgG molecules. Folding speed might be crucial to improve secretion, but addition of chaperones might have unpredictable effects to the balance between LC and HC, as their function might be fragment specific. Thus, such high-throughput screening methods as presented here are giving insights towards what is needed for effective engineering of multisubunit protein folding.

\section{Conclusions}

We show here that inducing an ER expansion in the yeast $S$. cerevisiae can improve the secretion capacity of full-length antibodies up to fourfold. In addition, six genes (PDI1, ERO1, KAR2, LSH1, SIL1, and CPR5) were expressed in order to improve the folding environment in the ER. Improving the folding environment by overexpression of ER folding factors is not always achieved in a predictable manner. However, in general the effects of cell engineering on IgG titers are manifesting themselves most clearly at higher cultivation temperatures. Our data demonstrates that expression levels are crucial, especially when combining different factors within one strain, as these showed no synergistic effects in antibody titers. However, by using UPR controlled promoters, the folding environment dynamically responds to changes in ER load and expression levels are adjusted only when necessary, and this could provide a better approach for strain engineering.

The PPIase Cpr5p was shown to enhance antibody secretion the most. Combined with the ER expansion by $\Delta o p i 1$, the improved specific antibody yield of over 10-fold higher compared to wild-type was achieved in small scale conditions. Although the strain reached a lower cell density, even the absolute antibody titer was over fivefold higher than the wild-type strain. This makes the $\Delta o p i 1+C P R 5-\mathrm{P}_{\mathrm{GPD}}$ strain a very interesting candidate for further studies, as the combination of strain engineering with process optimization might develop $S$. cerevisiae systems into a viable alternative for current antibody producing platforms.

\section{Methods}

All used FastDigest-restriction enzymes Phusion HighFidelity DNA Polymerase were obtained from Thermo Fisher Scientific (Vantaa, Finland). All media components and reagents were obtained from Sigma-Aldrich (Helsinki, Finland), unless stated otherwise. Yeast nitrogen base without amino acids (YNB) was obtained from BD (Vantaa, Finland). All sequences of oligonucleotides are listed in Additional file 6.

\section{Yeast strains}

All $S$. cerevisiae yeast strains were derived from the parental strains W303 $\alpha\left(\right.$ ATCC $\left.^{\circledR} 208353^{\mathrm{TM}}\right)$. Two types of stable yeast strains were used in this study: W303 $\alpha$ and W303 $\alpha \Delta$ opil, both with IgG1-producing genes (heavy and light chains encoding the monoclonal antibody C2B8) integrated to HIS3-site of the genome. These IgG-producing W $303 \alpha$ and W $303 \alpha \Delta$ opil background strains are referred to in the manuscript as wt and $\Delta o p i 1$, respectively. The W $303 \alpha \Delta$ opi1 deletion strain was created as described in Hegemann and Heick [64]. A 
loxP-kanMX-loxP disruption module targeting the OPI1 gene was generated from pUG6 [65] with PCR, using oligonucleotides 3 and 4 . The cassette was transformed to W303 $\alpha$ and positive transformants were selected using YPD-agar media, supplemented with $200 \mu \mathrm{g} / \mathrm{mL}$ G418 (Sigma-Aldrich, Helsinki, Finland). Positive knockouts were verified with colony-PCR using oligonucleotides 5 and 6. Cre-recombinase was expressed from plasmid pSH47 [65] to remove the gene disruption cassette from the genome following the protocol presented in Hegemann and Heick [64].

The plasmid for the IgG gene integration was achieved as follows: a fragment from pEK5 plasmid [12], containing genes for heavy and light chain each under GAL1-promoter, was amplified with PCR by using the oligonucleotides 1 and 2 . These oligonucleotides contained complementary $5^{\prime}$ ends to the pRS303N-plasmid [66]. pRS303N was linearized with Ecl136II and the amplified DNA fragmented was joined with the vector as described in Koskela and Frey [67]. The $6.8 \mathrm{~kb}$ DraIII and Bsp119I fragment of the resulting pEK12 plasmid was transformed

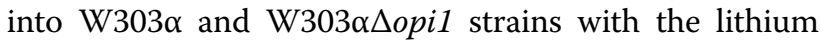
acetate method. Selection of transformed colonies was achieved by adding $200 \mu \mathrm{g} / \mathrm{mL}$ LEXSY NTC (Jena Bioscience, Jena, Germany) to the growth medium. IgG expression of the colonies was confirmed with ELISA.

\section{Yeast cultures for growth curves}

For recording of growth curves, cultures of exponentially growing cells were diluted to $\mathrm{OD}_{600}$ of 0.02 in YPD and YPGal (1 \% yeast extract, $2 \%$ peptone, and $2 \%$ glucose or galactose, respectively) and liquid synthetic drop out (SD) medium containing $2 \%$ raffinose or $2 \%$ raffinose supplemented with $2 \%$ galactose (SGal). One hundred microliters of these cultures were grown in a round-bottom microtiter plate, with continuous orbital shaking $(425 \mathrm{rpm}, 3 \mathrm{~mm})$ at $30{ }^{\circ} \mathrm{C}$ in an Eon Microplate Spectrophotometer (BioTek, Winooski, USA). $\mathrm{OD}_{600}$ measurements were taken every $15 \mathrm{~min}$ for $40 \mathrm{~h}$. The growth curves were analyzed with R-package grofit and the characteristic growth parameters were extracted from the spline fit, a data fitting function provided by the grofit-package [68]. The experiment was conducted with seven or eight culture replicates and the statistical significance of the difference between the two strains was determined with Wilcoxon signed rank test (as described below).

\section{Analysis of the UPR induction}

The UPR-responsive cassette encoding GFP from plasmid pDEP017 [35] was linearized with EcoRV and integrated into the TRP1-locus of $\triangle o p i 1$ and wt production strains.
The functionality of the integrated cassette was confirmed by induction of UPR through addition of $5 \mathrm{mM}$ DTT. For UPR measurements, the strains were grown over night at $30{ }^{\circ} \mathrm{C}, 250 \mathrm{rpm}$ and diluted to $\mathrm{OD}_{600}=0.1$ into 96-well plates using SGal media. Cultures were grown with continuous orbital shaking ( $425 \mathrm{rpm}, 3 \mathrm{~mm}$ ) at $30{ }^{\circ} \mathrm{C}$ using a Cytation 3 Microplate Spectrophotometer (BioTek, Winooski, USA), $\mathrm{OD}_{600}$ and GFP-fluorescence measurements were taken every $15 \mathrm{~min}$ for $24 \mathrm{~h}$.

\section{Cloning of overexpression plasmids}

The genomic DNAs of the selected genes were cloned into yeast shuttle vectors pRS415 and pRS416 containing TEF, GPD and GAL1 promoters $[69,70]$. The pRS41X vectors were modified to include the UPR-controlled promoters from S. cerevisiae genes PDI1 and KAR2. For exchanging of the promoter, fragments $355 \mathrm{bp}$ upstream of $P D I 1$ and 281 bp upstream of KAR2 were first amplified with PCR from yeast genomic DNA, using oligonucleotides 7, 8, 9 and 10 , respectively. Next, to generate complementary sequences overlapping with the vector backbone ends for insertion of these fragments with exonuclease and ligation-independent cloning (ELIC) [67], a second round of PCR was performed on these products with oligonucleotides 11, 12, 13 and 14, respectively. These fragments were inserted into $\mathrm{SacI} / \mathrm{Xba \textrm {I }}$ linearized plasmids pRS415 and pRS416 to create the $\mathrm{P}_{\mathrm{PDI} 1}$ and $\mathrm{P}_{\mathrm{KAR} 2}$ empty vectors with the different auxotrophic selection markers.

The plasmids expressing PDI1, ERO1, CPR5 and KAR2 under TEF, GPD and GAL1 promoters, were constructed using PCR on S. cerevisiae genomic DNA with their respective oligonucleotide pairs (oligonucleotides 15-22). The PCR amplified fragments were inserted into the SpeI XhoI sites of the pRS415 and pRS416 vectors, using standard ligation cloning.

The PDI1, ERO1, CPR5 and KAR2 plasmids with $\mathrm{P}_{\mathrm{KAR} 2}$ and $\mathrm{P}_{\mathrm{PDI} 1}$ promoters were constructed by using ELIC. A PCR reaction was made with oligo 36 ( $3^{\prime}$ end) and the oligo corresponding with the inserted gene from oligonucleotides $23-25$ ( $5^{\prime}$ end), and using the previously created TEF-promoter-gene plasmids as template. The newly generated fragments were used for a second round of PCR to add the complementary sequences for the vectors to the inserts, using oligo 36 ( $3^{\prime}$ end) with oligo 34 or 35 ( $5^{\prime}$ end). These final PCR products were inserted into the $\mathrm{SpeI} / \mathrm{XbaI}$ and $X h o I$ sites of the pRS-shuttle vectors using ELIC.

To construct all the LHS1 and SIL1 expression plasmids, oligonucleotides $26 \& 27$ and $28 \& 29$, respectively, were used to amplify the DNA of LHS1 and SIL1 from $S$. cerevisiae genomic DNA. In a second PCR round, the complementary sequences overlapping with the vectors were added to the inserts, using oligo 30 ( $3^{\prime}$ end) 
with either oligo $31,32,33,34$, or 35 ( $5^{\prime}$ end). These PCR products were inserted into the SpeI/XhoI and $\mathrm{XbaI} / \mathrm{XhoI}$ fragments of the pRS-shuttle vectors using ELIC cloning.

Empty marker plasmids for use as a control were the recirculated forms of pRS41X-vectors after SacI and NaeI digestion. A list of all plasmids used can be found in Additional file 7.

\section{Yeast expression cultures}

Screening of the yeast strains was conducted with 96deep well plates in a culture volume of $1 \mathrm{ml}$ per well. A colony from each strain was inoculated to SD medium lacking leucine and uracil, containing $2 \%$ raffinose. As a control, the IgG expressing wild-type and $\Delta$ opil strains with empty plasmids were included in every plate. Precultures were grown at $30^{\circ} \mathrm{C}, 250 \mathrm{rpm}$ for $21 \mathrm{~h}$. The culture was diluted 1:4 with fresh SD media, containing $2 \%$ raffinose, supplemented with $0.05 \mathrm{mg} / \mathrm{ml} \mathrm{BSA}$. The cultures for IgG expression were grown at $30{ }^{\circ} \mathrm{C}, 250 \mathrm{rpm}$ ) for $5.5 \mathrm{~h}$ before protein expression was induced by adding 0.5 or $2 \%$ galactose. Protein expression was continued for $24 \mathrm{~h}$ at 20,25 or $30{ }^{\circ} \mathrm{C}, 250 \mathrm{rpm}$. Culture deep-well plates were centrifuged at $2250 \mathrm{rcf}, 10 \mathrm{~min}$ to clarify the supernatant samples. Cell-free supernatant was divided into two $300 \mu \mathrm{l}$ samples adjusted to $1 \mathrm{x}$ PBT (PBS $\left(135 \mathrm{mM} \mathrm{NaCl}, 2.5 \mathrm{mM} \mathrm{KCl}, 10 \mathrm{mM} \mathrm{Na}_{2} \mathrm{HPO}_{4}, 1.75 \mathrm{mM}\right.$ $\left.\mathrm{KH}_{2} \mathrm{PO}_{4}\right)+0.5 \%$ Tween-20) and stored at $-20{ }^{\circ} \mathrm{C}$ until analysis, done within 2 days. Each sample was measured with three independent biological replicates, each with two technical replicates in ELISA. $\mathrm{OD}_{600}$ was measured before dilution and from final samples with an Eon Microplate Spectrophotometer (BioTek, Winooski, USA).

\section{Enzyme-linked immunosorbent assay (ELISA) for antibody titer measurement}

The ELISA assay was realized on MICROLAB STAR Liquid Handling workstation (Hamilton, Bonaduz, Switzerland) extended with ELx405 Select deep well washer (BioTek Winooski, USA) and Synergy 2 plate reader (BioTek Winooski, USA). Antibody titer was determined as described in de Ruijter and Frey [12].

\section{Data processing and statistical analysis}

All measurement data was collected, combined and moved into $R$ software environment and processed and visualized with the packages therein. Outliers resulting from measurement errors were manually removed after visual analysis of replicate measurements. The performance of the strains were validated with frequency analysis, where the count and relative frequency inside the percentile was determined by strain and for individual measurement points. The cut-off values for 5 th and 95th percentiles were determined with quantile () -function. P values are from independent two-group and two-tailed Wilcoxon signed rank test (performed with wilcox.test() -function), where the measurement values of the respective strain background standard served as the reference. The linear regression plane was fitted to the data with the function $\operatorname{lm}()$ where dependency of normalized antibody titers on the joint effects of normalized $\mathrm{OD}_{600}$ and normalized specific product yield was modeled.

\section{Real-time PCR}

Cultivation was conducted as described for screening assay: Selected strains were inoculated to $1 \mathrm{ml}$ media in 96-deep well plates grown once to saturation and diluted and grown for $5.5 \mathrm{~h}$. After induction with $2 \%$ galactose, the cultures were grown for 2 or $10 \mathrm{~h}$ at $30^{\circ} \mathrm{C} .1 .5 \mathrm{OD}_{600}$ of cells was collected for mRNA isolation. IgG expression was confirmed from supernatants with ELISA. mRNA was isolated from cell pellets with RNeasy ${ }^{\circledR}$ Mini Kit (Qiagen), and approximately $10 \mu \mathrm{g}$ of mRNA was treated with TURBO DNA-free ${ }^{\mathrm{TM}}$ DNAse $\left(\right.$ Ambion $^{\circledR}$ RNA by Life Technologies). After DNAse inactivation, mRNA was converted to cDNA with AffinityScript QPCR cDNA synthesis kit (Agilent Technologies, USA), according to manufacturer's protocol. qPCR was performed with a CFXConnect thermocycler (Bio-Rad, USA) on $100 \mathrm{ng}$ of cDNA using commercial PrimePCR ${ }^{\mathrm{TM}} \mathrm{SYBR}^{\circledR}$ Green Assay CPR5, Yeast primers (qSceCED0004208, Bio-Rad, USA), while $A C T 1$ levels were measured as a loading reference with primers 37 and 38. Maxima SYBR green/ fluorescin qPCR Master Mix (Thermo Scientific, USA) was used as reagent according to manufacturer's protocol. Results from three technical replicates were analyzed with the Bio-Rad CFX Manager 3.0 software, using ACT1 as loading control and with normalization to the CPR5 signal from the wt sample.

\section{Cellular clearance assay}

The cellular clearance assays was performed as described in De Ruijter and Frey [12]. Using anti-human IgG (Fc specific)-peroxidase labelled antibody produced in goat for heavy chain detection and anti-Human kappa light chains (Bound and Free)-peroxidase antibody produced in goat for light chain detection. Rabbit anti-tubulin antibody [EPR13799] (Abcam, Cambridge, UK) and goat anti-rabbit IgG (Fc specific)-peroxidase labelled were used for tubulin detection. Signal detection was done with the Supersignal ${ }^{\mathrm{TM}}$ West Pico Chemiluminescent Substrate (Thermo Fisher Scientific, Vantaa, Finland), following the manufacturer's instructions. 


\section{Additional files}

Additional file 1. Results from the screening process for all the strains with single gene additions, divided by the different experimental conditions. Values without defined gene or promoter are the strain background measurements for each conditions. The normalization of samples is always done to the background strain values from the same condition. Specific product yield is defined as antibody titer (denoted [AB]) divided by $\mathrm{OD}_{600^{-}}$ value. SD is an abbreviation for standard deviation. The values are means from three biological and two technical replicates.

Additional file 2. Effects of single folding factor overexpression on antibody secretion in wt and $\triangle$ opil strains at $2 \%$ galactose induction. A heatmap illustrating the effects that the different individual elements had on specific product yields in wt (A) and the $\triangle$ opil strain (B) backgrounds at $2 \%$ induction at $30^{\circ} \mathrm{C}$. The numbers represent the fold-changes that are calculated by normalizing to the respective mean specific product yields of the wt and $\triangle$ opil background strains.

Additional file 3. Screening results for all the strains with double gene additions grouped by strain background, temperature and induction strength. First, the values for the background strain standards are listed, to which the normalization is done always using the reference from the same condition. $[\mathrm{AB}]$ stands for antibody titer and SD for standard deviation and specific product yield is defined as $[\mathrm{AB}] / \mathrm{OD}_{600}$. The values are means from three biological and two technical replicates.

Additional file 4. Overview of the mean fold-changes for all the strains with double gene additions. For each analyzed gene pair the mean foldchanges in $\mathrm{OD}_{600}$, in Ig titer, and in specific product yield are shown. Averages were calculated from fold-changes that were first compared to the respective wild-type samples.

Additional file 5. Result from the frequency analysis showing the strains in the 95th and 5th percentile of individual measurements of specific product yield in each temperature and strain background. To determine which strains were enriched among the highest and lowest values of specific product yield, we conducted a frequency analysis for each strain background, separately for each temperature. Frequency represents the absolute count of measurements from the strain inside the percentile and the relative frequency is the frequency normalized to the total count of measurements in the percentile. P values were calculated with Wilcoxon signed rank test using the respective background strain measurements in the same temperature as the reference.

Additional file 6. List of oligonucleotides used in this study. All sequences of oligonucleotides used in this work are shown. Oligonucleotides 1-6 are for DNA integration into the yeast genome and for its confirmation. 7-14 were used for placement of $\mathrm{P}_{\mathrm{PDI} 11}$ and $\mathrm{P}_{\mathrm{KAR} 2}$ into the vector backbone, 15-36 were used for generation of the overexpression plasmids of the various folding factors. Finally 37 and 38 were used for ACT1 qPCR.

Additional file 7. List of all the plasmids in our overexpression library. Overview of all ER-resident protein expression plasmids used in this study. Original name, promoter, expressed gene and the corresponding auxotrophic marker are mentioned. All plasmids contain the cyc1 terminator.

\section{Authors' contributions}

$J R$ carried out the molecular cloning work, performed the screening and cellular clearance experiments, contributed to the mRNA experiments, and drafted the manuscript. EK created the background yeast strains, contributed to molecular cloning and the screening experiments, carried out the mRNA experiments, the statistical analysis, and drafted the manuscript. AF conceived the study, participated in its design, and helped to draft the manuscript. All authors read and approved the final manuscript.

\section{Acknowledgements}

The work was supported by Aalto University Bioeconomy facilities and Aalto University School of Chemical Technology. EV Koskela is a recipient of a doctoral study Grant from the School of Chemical Technology, Aalto University.
The authors would like to acknowledge Dr. András Pásztor (Aalto University, Finland) for making Fig. 2. We thank Olli Koskela for insights in statistics and Dr. David Pincus for kindly providing the pDEP017 plasmid.

\section{Competing interests}

The authors declare that they have no competing interests.

Received: 15 November 2015 Accepted: 11 May 2016

Published online: 23 May 2016

\section{References}

1. Mattanovich D, Sauer M, Gasser B. Yeast biotechnology: teaching the old dog new tricks. Microb Cell Fact. 2014;13:34.

2. Ferrer-Miralles N, Domingo-Espín J, Corchero JL, Vázquez E, Villaverde A. Microbial factories for recombinant pharmaceuticals. Microb Cell Fact. 2009:8:17.

3. Piirainen MA, Boer H, de Ruijter JC, Frey AD. A dual approach for improving homogeneity of a human-type $\mathrm{N}$-glycan structure in Saccharomyces cerevisiae. Glycoconj J. 2016;33:189-99.

4. Parsaie Nasab F, Aebi M, Bernhard G, Frey AD. A combined system for engineering glycosylation efficiency and glycan structure in Saccharomyces cerevisiae. Appl Environ Microbiol. 2013;79:997-1007.

5. I Iiris A, Tohda H, Kumagai H, Takegawa K. Engineering of protein secretion in yeast: strategies and impact on protein production. Appl Microbiol Biotechnol. 2010;86:403-17.

6. Hou J, Tyo KEJ, Liu Z, Petranovic D, Nielsen J. Metabolic engineering of recombinant protein secretion by Saccharomyces cerevisiae. FEMS Yeast Res. 2012;12:491-510.

7. Mattanovich D, Gasser B, Hohenblum H, Sauer M. Stress in recombinant protein producing yeasts. J Biotechnol. 2004;113:121-35.

8. Snapp EL. Unfolded protein responses with or without unfolded proteins? Cells. 2012;1:926-50.

9. Friedlander R, Jarosch E, Urban J, Volkwein C, Sommer T. A regulatory link between ER-associated protein degradation and the unfolded-protein response. Nat Cell Biol. 2000;2:379-84.

10. Travers KJ, Patil CK, Wodicka L, Lockhart DJ, Weissman JS, Walter P. Functional and genomic analyses reveal an essential coordination between the unfolded protein response and ER-associated degradation. Cell. 2000;101:249-58.

11. Valkonen M, Penttilä M, Saloheimo M. Effects of inactivation and constitutive expression of the unfolded- protein response pathway on protein production in the yeast Saccharomyces cerevisiae. Appl Environ Microbiol. 2003;69:2065-72.

12. de Ruijter JC, Frey AD. Analysis of antibody production in Saccharomyces cerevisiae: effects of ER protein quality control disruption. Appl Microbiol Biotechnol. 2015:99:9061-71.

13. Thibault G, Ismail N, Ng DTW. The unfolded protein response supports cellular robustness as a broad-spectrum compensatory pathway. Proc Natl Acad Sci USA. 2011;108:20597-602.

14. Cox JS, Chapman RE, Walter P. The unfolded protein response coordinates the production of endoplasmic reticulum protein and endoplasmic reticulum membrane. Mol Biol Cell. 1997;8:1805-14.

15. Schuck S, Prinz WA, Thorn KS, Voss C, Walter P. Membrane expansion alleviates endoplasmic reticulum stress independently of the unfolded protein response. J Cell Biol. 2009;187:525-36.

16. Federovitch CM, Ron D, Hampton RY. The dynamic ER: experimental approaches and current questions. Curr Opin Cell Biol. 2005;17:409-14.

17. van Anken E, Braakman I. Endoplasmic reticulum stress and the making of a professional secretory cell. Crit Rev Biochem Mol Biol. 2005;40:269-83.

18. van Anken $E$, Braakman I. Versatility of the endoplasmic reticulum protein folding factory. Crit Rev Biochem Mol Biol. 2005;40:191-228.

19. Christis C, Fullaondo A, Schildknegt D, Mkrtchian S, Heck AJR, Braakman I. Regulated increase in folding capacity prevents unfolded protein stress in the ER. J Cell Sci. 2010;123(Pt 5):787-94.

20. van Anken E, Romijn EP, Maggioni C, Mezghrani A, Sitia R, Braakman I, Heck AJR. Sequential waves of functionally related proteins are expressed when B cells prepare for antibody secretion. Immunity. 2003;18:243-53. 
21. Feige MJ, Hendershot LM, Buchner J. How antibodies fold. Trends Biochem Sci. 2010;35:189-98.

22. Braakman I, Bulleid NJ. Protein folding and modification in the mammalian endoplasmic reticulum. Annu Rev Biochem. 2011;80:71-99.

23. Jonikas MC, Collins SR, Denic V, Oh E, Quan EM, Schmid V, Weibezahn J, Schwappach B, Walter P, Weissman JS, Schuldiner M. Comprehensive characterization of genes required for protein folding in the endoplasmic reticulum. Science. 2009;323:1693-7.

24. Meunier L, Usherwood YK, Chung KT, Hendershot LM. A subset of chaperones and folding enzymes form multiprotein complexes in endoplasmic reticulum to bind nascent proteins. Mol Biol Cell. 2002;13:4456-69.

25. Jansen G, Määttänen P, Denisov AY, Scarffe L, Schade B, Balghi H, Dejgaard K, Chen LY, Muller WJ, Gehring K, Thomas DY. An interaction map of endoplasmic reticulum chaperones and foldases. Mol Cell Proteomics. 2012;11:710-23.

26. Shusta EV, Raines RT, Plückthun A, Wittrup KD. Increasing the secretory capacity of Saccharomyces cerevisiae for production of single-chain antibody fragments. Nat Biotechnol. 1998;16:773-7.

27. Hackel BJ, Huang D, Bubolz JC, Wang XX, Shusta EV. Production of soluble and active transferrin receptor-targeting single-chain antibody using Saccharomyces cerevisiae. Pharm Res. 2006;23:790-7.

28. Xu P, Raden D, Doyle FJ, Robinson AS. Analysis of unfolded protein response during single-chain antibody expression in Saccaromyces cerevisiae reveals different roles for BiP and PDI in folding. Metab Eng. 2005;7:269-79.

29. Chumnanpuen P, Nookaew I, Nielsen J. Integrated analysis, transcriptome-lipidome, reveals the effects of INO-level (INO2 and INO4) on lipid metabolism in yeast. BMC Syst Biol. 2013;7(Suppl 3):S7.

30. Carman GM, Han G-S. Regulation of phospholipid synthesis in yeast. J Lipid Res. 2008;50(Supplement):S69-73.

31. Chen M, Hancock LC, Lopes JM. Transcriptional regulation of yeast phospholipid biosynthetic genes. Biochim Biophys Acta. 2007;1771:310-21.

32. Cenci S, Sitia R. Managing and exploiting stress in the antibody factory. FEBS Lett. 2007;581:3652-7.

33. Gass J. Stressed-out B cells? Plasma-cell differentiation and the unfolded protein response. Trends Immunol. 2004;25:17-24.

34. Guerfal M, Claes K, Knittelfelder O, De Rycke R, Kohlwein SD, Callewaert $\mathrm{N}$. Enhanced membrane protein expression by engineering increased intracellular membrane production. Microb Cell Fact. 2013;12:122.

35. Pincus D, Chevalier MW, Aragón T, van Anken E, Vidal SE, El-Samad H, Walter P. BiP binding to the ER-stress sensor Ire1 tunes the homeostatic behavior of the unfolded protein response. PLoS Biol. 2010;8:e1000415.

36. Mori K, Ogawa N, Kawahara T, Yanagi H, Yura T. Palindrome with spacer of one nucleotide is characteristic of the cis-acting unfolded protein response element in Saccharomyces cerevisiae. J Biol Chem. 1998;273:9912-20.

37. Ugolini S, Tosato V, Bruschi CV. Selective fitness of four episomal shuttlevectors carrying HIS3, LEU2, TRP1, and URA3 selectable markers in SaCcharomyces cerevisiae. Plasmid. 2002;47:94-107.

38. Frigerio G, Pelham HR. A Saccharomyces cerevisiae cyclophilin resident in the endoplasmic reticulum. J Mol Biol. 1993;233:183-8.

39. Lilie $H$, Rudolph $\mathrm{R}$, Buchner J. Association of antibody chains at different stages of folding: prolyl isomerization occurs after formation of quaternary structure. J Mol Biol. 1995;248:190-201.

40. Thies MJ, Mayer J, Augustine JG, Frederick CA, Lilie H, Buchner J. Folding and association of the antibody domain $\mathrm{CH} 3$ : prolyl isomerization preceeds dimerization. J Mol Biol. 1999;293:67-79.

41. Feige MJ, Walter S, Buchner J. Folding mechanism of the $\mathrm{CH} 2$ antibody domain. J Mol Biol. 2004;344:107-18.

42. Hale SJ, Lovell SC, de Keyzer J, Stirling CJ. Interactions between Kar2p and its nucleotide exchange factors Sil $1 \mathrm{p}$ and Lhs1 $\mathrm{p}$ are mechanistically distinct. J Biol Chem. 2010;285:21600-6.

43. Steel GJ, Fullerton DM, Tyson JR, Stirling CJ. Coordinated activation of Hsp70 chaperones. Science. 2004;303:98-101.

44. Payne T, Finnis C, Evans LR, Mead DJ, Avery SV, Archer DB, Sleep D. Modulation of chaperone gene expression in mutagenized Saccharomyces cerevisiae strains developed for recombinant human albumin production results in increased production of multiple heterologous proteins. Appl Environ Microbiol. 2008;74:7759-66.

45. Xu L, Shen Y, Hou J, Peng B, Tang H, Bao X. Secretory pathway engineering enhances secretion of cellobiohydrolase I from Trichoderma reesei in Saccharomyces cerevisiae. J Biosci Bioeng. 2014;117:45-52.
46. Robinson AS, Hines V, Wittrup KD. Protein disulfide isomerase overexpression increases secretion of foreign proteins in Saccharomyces cerevisiae. Biotechnology (NY). 1994;12:381-4.

47. Kim EJ, Park YK, Lim HK, Park YC, Seo JH. Expression of hepatitis B surface antigen $\mathrm{S}$ domain in recombinant Saccharomyces cerevisiae using GAL 1 promoter. J Biotechnol. 2009;141:155-9.

48. Delic M, Göngrich R, Mattanovich D, Gasser B. Engineering of protein folding and secretion-strategies to overcome bottlenecks for efficient production of recombinant proteins. Antioxid Redox Signal. 2014;21:414-37.

49. Grubb S, Guo L, Fisher EA, Brodsky JL. Protein disulfide isomerases contribute differentially to the endoplasmic reticulum-associated degradation of apolipoprotein B and other substrates. Mol Biol Cell. 2012;23:520-32.

50. Kimata Y, Kimata YI, Shimizu Y, Abe H, Farcasanu IC, Takeuchi M, Rose MD, Kohno K. Genetic evidence for a role of BiP/Kar2 that regulates Ire1 in response to accumulation of unfolded proteins. Mol Biol Cell. 2003;14:2559-69.

51. Bertolotti A, Zhang Y, Hendershot LM, Harding HP, Ron D. Dynamic interaction of BiP and ER stress transducers in the unfolded-protein response. Nat Cell Biol. 2000;2:326-32.

52. Feizi A, Österlund T, Petranovic D, Bordel S, Nielsen J. Genome-scale modeling of the protein secretory machinery in yeast. PLoS One. 2013;8:e63284

53. Chillarón J, Haas IG. Dissociation from BiP and retrotranslocation of unassembled immunoglobulin light chains are tightly coupled to proteasome activity. Mol Biol Cell. 2000;11:217-26.

54. Vanhove M, Usherwood YK, Hendershot LM. Unassembled Ig heavy chains do not cycle from BiP in vivo but require light chains to trigger their release. Immunity. 2001;15:105-14.

55. Kazemi Seresht A, Cruz AL, de Hulster E, Hebly M, Palmqvist EA, van Gulik W, Daran JM, Pronk J, Olsson L. Long-term adaptation of Saccharomyces cerevisiae to the burden of recombinant insulin production. Biotechnol Bioeng. 2013;110:2749-63.

56. Gonzalez R, Andrews BA, Molitor J, Asenjo JA. Metabolic analysis of the synthesis of high levels of intracellular human SOD in Saccharomyces cerevisiae rhSOD 2060411 SGA122. Biotechnol Bioeng. 2003;82:152-69.

57. Görgens JF, van Zyl WH, Knoetze JH, Hahn-Hägerdal B. The metabolic burden of the $P G K 1$ and $A D H 2$ promoter systems for heterologous xylanase production by Saccharomyces cerevisiae in defined medium. Biotechnol Bioeng. 2001;73:238-45.

58. Dietmair S, Hodson MP, Quek L-E, Timmins NE, Gray P, Nielsen LK. A multiomics analysis of recombinant protein production in Hek293 cells. PLoS One. 2012;7:e43394.

59. Yamano N, Takahashi M, Ali Haghparast SM, Onitsuka M, Kumamoto T, Frank J, Omasa T. Increased recombinant protein production owing to expanded opportunities for vector integration in high chromosome number Chinese hamster ovary cells. J Biosci Bioeng. 2016. doi:10.1016/j. jbiosc.2016.01.002.

60. Seo JS, Kim YJ, Cho JM, Baek E, Lee GM. Effect of culture pH on recombinant antibody production by a new human cell line, F2N78, grown in suspension at 33.0 and $\# \times B 0 ; C$ and 37.0 and \#xBO;C. Appl Microbiol Biotechnol. 2013;97:5283-91.

61. Maccani A, Landes N, Stadlmayr G, Maresch D, Leitner C, Maurer M, Gasser B, Ernst W, Kunert R, Mattanovich D. Pichia pastoris secretes recombinant proteins less efficiently than Chinese hamster ovary cells but allows higher space-time yields for less complex proteins. Biotechnol J. 2014;9:526-37.

62. Edros RZ, McDonnell S, Al-Rubeai M. Using molecular markers to characterize productivity in Chinese hamster ovary cell lines. PLoS One. 2013;8:e75935.

63. Borth N, Strutzenberger K, Kunert R, Steinfellner W, Katinger $\mathrm{H}$. Analysis of changes during subclone development and ageing of human antibodyproducing heterohybridoma cells by northern blot and flow cytometry. J Biotechnol. 1999;67:57-66.

64. Hegemann JH, Heick SB. Delete and repeat: a comprehensive toolkit for sequential gene knockout in the budding yeast Saccharomyces cerevisiae. Methods Mol Biol. 2011;765:189-206.

65. Güldener U, Heck S, Fielder T, Beinhauer J, Hegemann JH. A new efficient gene disruption cassette for repeated use in budding yeast. Nucleic Acids Res. 1996;24:2519-24. 
66. Taxis C, Knop M. System of centromeric, episomal, and integrative vectors based on drug resistance markers for Saccharomyces cerevisiae. Biotechniques. 2006;40:73-8.

67. Koskela EV, Frey AD. Homologous recombinatorial cloning without the creation of single-stranded ends: exonuclease and ligation-independent cloning (ELIC). Mol Biotechnol. 2015;57:233-40.

68. Kahm M, Hasenbrink G, Lichtenberg-Fraté H, Ludwig J, Kschischo M. grofit : fitting Biological growth curves with R. J Stat Softw. 2010;33:1-21.
69. Mumberg D, Müller R, Funk M. Yeast vectors for the controlled expression of heterologous proteins in different genetic backgrounds. Gene. 1995; 156:119-22.

70. Mumberg D, Müller R, Funk M. Regulatable promoters of Saccharomyces cerevisiae: comparison of transcriptional activity and their use for heterologous expression. Nucleic Acids Res. 1994;22:5767-8. 